\title{
Unterrichtsqualität in den Fachdidaktiken im deutschsprachigen Raum - zwischen Generik und Fachspezifik
}

\author{
Anna-Katharina Praetorius (D) C Christian Herrmann (D) \\ Erin Gerlach $\mathbb{D} \cdot$ Meik Zülsdorf-Kersting • Benjamin Heinitz $(\mathbb{D})$ \\ Andreas Nehring (D)
}

Eingegangen: 21. August 2019 / Überarbeitet: 19. Juni 2020 / Angenommen: 22. Juni 2020 / Online publiziert: 7. August 2020

(C) Der/die Autor(en) 2020

Zusammenfassung Im vorliegenden Diskussionsbeitrag wird der Forschungsstand zu Unterrichtsqualität für verschiedene Fachdidaktiken - naturwissenschaftliche Fächer, Sport und Geschichte - vor dem Hintergrund der allgemeinen empirischen Unterrichtsforschung vergleichend diskutiert. Als Vergleichsgrundlage wurde ein Framework mit den sieben Dimensionen Auswahl und Thematisierung von Inhalten und Fachmethoden, kognitive Aktivierung, Unterstützung des Übens, formatives Assessment, Unterstützung des Lernens aller Schülerinnen und Schüler, sozio-emotionale Unterstützung und Klassenführung genutzt (Praetorius und Charalambous 2018). Es zeigte sich, dass die Fachspezifität von der fokussierten Ebene abhing: Während eine Ergänzung des Frameworks auf Ebene der sieben Dimensionen nicht notwendig erschien, waren auf der Ebene der Subdimensionen und insbesondere der diesen Subdimensionen zugeordneten beobachtbaren Indikatoren fachspezifische Anpassungen und Ergänzungen erforderlich. Der Beitrag schließt mit einer Diskussion zentraler Herausforderungen für zukünftige Forschung.

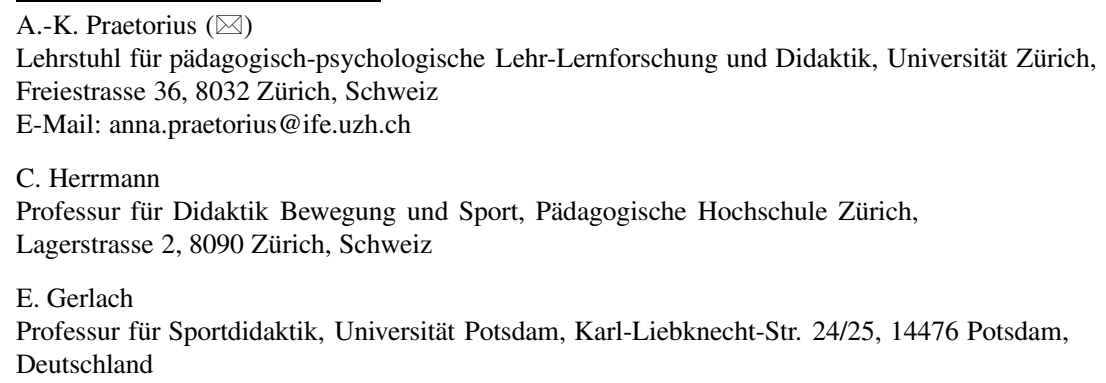


Schlüsselwörter Unterrichtsqualität · Fachdidaktiken · Synthese · Sport · Naturwissenschaften · Geschichte

\title{
Teaching quality in different subject matters in German-speaking countries-Inbetween genericness and subject-specificity
}

\begin{abstract}
In this synthesis paper, the current state of research regarding teaching quality is discussed comparing science education, physical education, and history education, by referring to research on teaching in general. A framework with seven dimensions (Praetorius and Charalambous 2018) was used as a comparison base. This framework contains the dimensions of selecting and introducing the content and subject matter methods, cognitive activation, support of practicing, formative assessment, support of all students' learning, socio-emotional support, and classroom management. The comparison across subject matters showed that subject-specificity was dependent on the level under consideration: On the dimension level, no adaptations and supplementations were deemed necessary. On the level of sub-dimensions, and especially on the level of observable indicators for these sub-dimensions, this was, however, the case. Challenges for future research are discussed.
\end{abstract}

Keywords Teaching quality - Instructional quality · Subject matters - Synthesis · Physical education $\cdot$ Science education $\cdot$ History education

Die Frage, was die Qualität von Unterricht ausmacht, beschäftigt die empirische Unterrichtsforschung seit Jahrzehnten (für eine Übersicht siehe Helmke 2017). Dabei wurden in den letzten Jahren in unterschiedlichen Forschungsgruppen verschiedene Konzeptualisierungen zur Beschreibung von Unterrichtsqualität entwickelt, die seitdem parallel eingesetzt werden. Diese unterscheiden sich darin, welche Dimensionen von Unterrichtsqualität einbezogen werden, wie diese strukturiert und benannt sind und danach, ob sie ausschließlich generisch, d.h. fachübergreifend, ausgelegte Dimensionen in den Blick nehmen (z.B. drei Basisdimensionen, vgl. Klieme et al. 2001) oder auch fachspezifische Dimensionen von Unterrichtsqualität (z. B. für die Mathematik, vgl. Learning Mathematics for Teaching Project 2011). Eine aktuelle Synthese solcher Konzeptualisierungen für den Mathematikunterricht (Praetorius und Charalambous 2018) deutet darauf hin, dass Unterrichtsqualität mittels der im deutschsprachigen Raum populären, generisch ausgerichteten drei Basisdimensionen nicht umfassend beschrieben werden kann (Klassenführung, konstruktive Unterstützung, kognitive Aktivierung; siehe Beitrag 1, Praetorius, Rogh und Kleickmann, in diesem Thementeil), sondern mathematikdidaktischer Ergänzungen bedarf. Allerdings bezieht sich diese Feststellung lediglich auf das Fach Mathematik. Die Fokussierung auf den Mathematikunterricht trifft auch auf einen Großteil der bisherigen Studien in der generisch ausgerichteten empirischen Unterrichtsforschung zu. Die Diskurse um Unterrichtsqualität in anderen Fachdidaktiken werden vielfach unverbunden dazu geführt. Die im Thementeil für die kontrastierend ausgewählten 
Fachdidaktiken der naturwissenschaftlichen Fächer ${ }^{1}$, Sport und Geschichte aufgearbeiteten Forschungsstände veranschaulichen die Notwendigkeit, den Blick über den Mathematikunterricht hinaus zu weiten (siehe Beiträge 2-4 in diesem Thementeil: Heinitz \& Nehring; Herrmann \& Gerlach; Zülsdorf-Kersting). Die Auswahl der Fächer erfolgte dabei so, dass durch die Zugehörigkeit zu unterschiedlichen Bereichen - Naturwissenschaften (mit besonderem Fokus auf Chemie), Gesellschaftswissenschaften (Geschichte) und dem musisch-ästhetischen Bereich (Sport) - eine möglichst große Bandbreite unterschiedlicher Fächer abgedeckt werden konnte.

Im vorliegenden Beitrag werden die zentralen Ergebnisse der Einzelbeiträge des Thementeils vergleichend diskutiert. Das methodische Vorgehen in den Einzelbeiträgen wie auch die herangezogenen Forschungsarbeiten wurden in den Einzelbeiträgen umfassend dargestellt und werden an dieser Stelle nur wiederholt, wenn es das Textverständnis erfordert. Wir gehen zunächst auf die Relevanz eines Vergleichs zwischen verschiedenen Fächern sowie der allgemeinen empirischen Unterrichtsforschung ein und leiten daraus die Fragestellungen des Beitrags ab (Abschn. 1). Danach werden der gewählte methodische Ansatz (Abschn. 2) sowie die Ergebnisse des Vergleichs der fachdidaktischen Diskurse zu Unterrichtsqualität dargestellt (Abschn. 3) und diskutiert (Abschn. 4).

\section{Relevanz und Fragestellungen}

In der empirischen Unterrichtsforschung wurden in den letzten Jahrzehnten insbesondere generische Dimensionen von Unterrichtsqualität mit dem Ziel der Identifikation allgemeingültiger Aspekte von Unterrichtsqualität fokussiert (Helmke 2017; Kunter und Trautwein 2013). Unterrichtsqualität wird dabei im Sinne des Educational Effectiveness Paradigmas vornehmlich über die Effektivität von Unterricht bestimmt (z. B. Muijs et al. 2014; Seidel und Shavelson 2007). Unterricht ist demzufolge dann als qualitätsvoll zu bezeichnen, wenn er erwünschte Entwicklungen bei Schülerinnen und Schülern (z. B. Leistungszuwachs) wahrscheinlich macht. Die konkreten empirischen Untersuchungen fokussierten dabei generische Merkmale von Unterrichtsqualität, fanden aber vorrangig im Mathematikunterricht sowie in naturwissenschaftlichen Fächern statt (siehe z. B. Kunter und Ewald 2016; Praetorius et al. 2018).

In den Folgejahren wurde insbesondere von Mathematikdidaktikerinnen und -didaktikern, aber auch von anderen Fachdidaktikerinnen und -didaktikern, vermehrt darauf hingewiesen, dass zentrale Aspekte von Unterrichtsqualität mit den bislang vorrangig generisch ausgerichteten Dimensionen nicht hinreichend erfasst werden. Die Notwendigkeit einer Anpassung und Ergänzung um fachspezifische Unterrichtsaspekte wird u. a. damit begründet, dass insbesondere diese fachlichen Aspekte von Unterricht lernwirksam sind (Seidel und Shavelson 2007). In der Folge wurden neben generisch ausgerichteten Instrumenten zur Erfassung von Unterrichtsqualität

\footnotetext{
1 Wenn im Folgenden von den naturwissenschaftlichen Fächern oder den Fachdidaktiken der Naturwissenschaften die Rede ist, so sind damit die Fachdidaktiken der Fächer Biologie, Chemie, Physik sowie die naturwissenschaftlichen Anteile des Sachunterrichts gemeint.
} 
stärker fachspezifische Instrumente entwickelt. Einige dieser Instrumente umfassen dabei ausschließlich bedeutsame Dimensionen von Unterrichtsqualität aus mathematikdidaktischer Sicht (z.B. MQI, vgl. Charalambous und Litke 2018), weitere hybride Instrumente kombinieren generische und mathematikspezifische Dimensionen (z.B. TRU, vgl. Schoenfeld 2018). Forschungsgruppen unterscheiden sich danach, welches der Instrumente sie einsetzen. Sie treffen damit auch eine Auswahl hinsichtlich der fokussierten Dimensionen von Unterrichtsqualität sowie eine Wahl hinsichtlich deren Strukturierung und Benennung. Praetorius und Charalambous (2018) haben eine umfassende konzeptuelle Integration generischer und fachspezifischer Aspekte des Mathematikunterrichts aus zwölf im deutschsprachigen Raum sowie international eingesetzten Instrumenten vorgelegt (generische Frameworks: CLASS, DMEE, ISTOF, 3 Basisdimensionen; mathematikspezifische Frameworks: IQA, MQI, M-Scan; hybride generisch-mathematikspezifische Frameworks: TRU, UTOP, TEDS-Unterricht, MECORS \& QoT). In der Folge eines induktiven, konsensuellen Vorgehens zur Identifikation gemeinsamer und spezifischer Dimensionen, Subdimensionen sowie beobachtbarer Indikatoren konnte ein Framework zusammengestellt werden (siehe Spalte 1 in Tab. 1; siehe auch Praetorius et al. in diesem Thementeil). Das im Folgenden als Syntheseframework bezeichnete Framework fasst generische und fachspezifische Aspekte von Unterrichtsqualität mit dem Ziel einer umfassenden Erfassung zusammen.

Vier der sieben identifizierten Dimensionen, ihre jeweiligen Subdimensionen sowie die diesen zugeordneten Indikatoren fokussieren auf eine prototypische LehrLernsequenz: (1) Eine angemessene Auswahl und Thematisierung von Inhalten und Fachmethoden ${ }^{2}$ ermöglicht die Auseinandersetzung mit relevanten Lerngegenständen; (2) die kognitive Aktivierung ist ausgerichtet auf die Unterstützung tiefenverarbeitender Lernprozesse; (3) die Unterstützung des Übens fokussiert auf die Konsolidierung des Gelernten und (4) formatives Assessment wird durchgeführt, um Informationen zur Optimierung von Lernprozessen zu erhalten. Die drei weiteren Dimensionen bilden die Grundlage für die Lehr-Lernsequenz: (5) Unterstïtzung des Lernens aller Schülerinnen und Schüler (z. B. Differenzierung und Adaptivität) sowie (6) sozio-emotionale Unterstützung sind für alle Schritte des Lernprozesses relevant, und (7) Klassenführung wird als grundlegende Voraussetzung angesehen, um Lernprozesse zu ermöglichen. Die vier Dimensionen, die sich an einzelne Schritte der Lehr-Lernsequenz anlehnen, umfassen dem Syntheseframework entsprechend sowohl generische als auch fachspezifische Aspekte, wobei insbesondere bei der Dimension Auswahl und Thematisierung von Inhalten und Fachmethoden eine stärkere Gewichtung fachspezifischer Aspekte erfolgte. Die drei weiteren, für alle Schritte im Lernprozess relevanten Dimensionen umfassen nahezu ausschließlich (Klassenführung und sozio-emotionale Unterstützung) oder mehrheitlich (Unterstützung des Lernens aller Schüler*innen) generische Aspekte.

Aufgrund des mathematikdidaktischen Fokus bleibt jedoch weitgehend offen, ob dieses Syntheseframework, das generische und mathematikdidaktische Dimensio-

\footnotetext{
${ }^{2}$ Die Benennung der Dimension wurde im Rahmen des vorliegenden Beitrags im Vergleich zur ursprünglichen Fassung bei Praetorius und Charalambous (2018) um die Auswahl und Thematisierung von Fachmethoden ergänzt (siehe auch Tab. 1).
} 
nen kombiniert, auch für andere Fächer Gültigkeit hat. Um diesem Desiderat zu begegnen, werden im vorliegenden Beitrag drei Fragestellungen bearbeitet:

1. Welche Überschneidungen existieren zwischen dem Syntheseframework und den fachspezifischen Merkmalskatalogen zu Unterrichtsqualität in den naturwissenschaftlichen Fächern sowie in den Fächern Sport und Geschichte?

2. Welche im Syntheseframework als relevant erachteten (Sub-)Dimensionen von Unterrichtsqualität werden in den fachdidaktischen Diskursen der naturwissenschaftlichen Fächer sowie der Fächer Sport und Geschichte nicht berücksichtigt?

3. Welche fachspezifischen Anpassungen und Ergänzungen des Syntheseframeworks sind nötig, um die fachspezifischen (Sub-)Dimensionen und Indikatoren von Unterrichtsqualität in den naturwissenschaftlichen Fächern sowie in den Fächern Sport und Geschichte abbilden zu können?

\section{Methode}

Die Bearbeitung der Fragestellungen erfolgte im gesamten interdisziplinären Team, so dass eine diskursive Aushandlung einerseits zwischen Vertreterinnen und Vertretern der einzelnen Fachdidaktiken sowie andererseits zwischen denjenigen der Fachdidaktiken und der allgemeinen empirischen Unterrichtsforschung ermöglicht wurde (insgesamt 6 Personen, davon 1-2 Vertreter*innen pro Fach bzw. Ausrichtung; zu den damit verbundenen Limitationen der Arbeit siehe Abschn. 4.4). Grundlage der vorliegenden Analyse bildete das im vorherigen Abschnitt beschriebene Syntheseframework (für eine ausführliche Herleitung und Darstellung siehe Praetorius et al. in diesem Thementeil). Es bietet den Vorteil, dass neben generischen auch fachspezifische (Sub-)Dimensionen enthalten sind (z.B. Akkuratheit der vermittelten Inhalte) und damit einige der notwendigen fachspezifischen Anpassungen und Ergänzungen bereits abgedeckt werden können.

Die Diskussionen im interdisziplinären Team orientierten sich an den Darstellungen in den Einzelbeiträgen dieses Heftes aus den verschiedenen Disziplinen. In diesen Beiträgen wurden die im Syntheseframework abgebildeten (Sub-)Dimensionen und Indikatoren mit den erarbeiteten Forschungsständen zu Unterrichtsqualität in den naturwissenschaftlichen Fächern sowie den Fächern Sport und Geschichte verglichen. ${ }^{3}$ Als Forschungsstand wurden dabei diejenigen Aspekte von Unterrichtsqualität einbezogen, die in dem jeweiligen Fach theoretisch als zentrale Aspekte angenommen werden und/oder empirisch untersucht wurden (Letzteres in Tab. 1 mit (E) gekennzeichnet). Die betreffenden empirischen Untersuchungen umfassen qualitative Befragungen, korrelative und varianzanalytische querschnittliche Untersuchungen, (videobasierte) Beobachtungsstudien, Interventionsstudien sowie längsschnittliche Untersuchungen.

\footnotetext{
3 Da der vorliegende Diskussionsbeitrag auf den fachspezifischen Beiträgen basiert, wird im Folgenden nur auf zentrale Literaturquellen verwiesen und in Tab. 1 sowie im Anhang eine Auflistung der Literatur der Literaturrecherche vorgenommen. Die gesamte herangezogene Literatur kann in den Literaturverzeichnissen der Einzelbeiträge nachgelesen werden. Für Details zum methodischen Vorgehen siehe ebenfalls die Einzelbeiträge.
} 
Um den aktuellen Forschungsstand möglichst umfassend abzudecken, wurde für jedes Fach in Anlehnung an das PRISMA-Statement (preferred reporting items for systematic reviews and meta- $a$ nalyses; Ziegler et al. 2011) eine systematische Literaturrecherche von Forschungsarbeiten im deutschsprachigen Raum durchgeführt. Forschungsarbeiten außerhalb des deutschsprachigen Raums wurden aufgrund der teils stark unterschiedlichen fachdidaktischen Konzeptionen in den verschiedenen Ländern und Sprachräumen nicht berücksichtigt (siehe auch Beiträge 2-4 in diesem Themenheft). Die gewählten Datenbanken und Suchbegriffe unterschieden sich je nach Fach/Fächergruppe. Dies liegt erstens an den unterschiedlichen Begrifflichkeiten, die in den jeweiligen fachspezifischen Diskursen Verwendung finden und somit zur Identifikation der relevanten Literatur als geeignet erschienen, zweitens an unterschiedlichen Verschlagwortungen der verwendeten Datenbanken und drittens an der Orientierung des Reviews für die Naturwissenschaften an einem bereits existierenden, international ausgerichteten Review.

Für das Fach Sport wurde neben der erziehungswissenschaftlich ausgerichteten Datenbank peDOCS die sportwissenschaftliche Datenbank bisp-surf.de verwendet. Die verwendeten Suchbegriffe lauteten „Unterrichtsqual*“, „Unterricht“ UND „qualität“, „gut*“ UND „Unterricht“, „Sportunterricht“ UND „Klassenführung“, ,Aktivierung“, „Klima“" sowie in peDOCS zusätzlich „Sport“. Nach dem Screening verblieben 42 relevante theoretisch-konzeptionelle sowie empirische Literaturquellen für die Analysen (vgl. Herrmann \& Gerlach in diesem Thementeil). Für das Fach Geschichte wurde neben peDOCS die geschichtsdidaktische Datenbank Index Didacticorum verwendet. Suchbegriffe bei peDOCS lauteten „Unterrichtsqualität“ UND „Geschichtsunterricht“ UND „gut“ sowie „,history“ UND „quality“, Suchbegriffe für die geschichtsdidaktische Datenbank waren „Forschung“ UND „Unterrichtsforschung“ UND „Unterrichtsangebot“ UND „Prozessqualität“ sowie „Forschung“ UND „Unterrichtsforschung“ UND „Wirkungen“. Nach dem Screening wurden 23 theoriebezogene sowie empirische Publikationen in die Analysen einbezogen (vgl. Zülsdorf-Kersting in diesem Thementeil). Für die Naturwissenschaften wurde aufgrund der hohen Anzahl an Studien im Bereich Unterrichtsqualität eine Fokussierung auf quantitative Videostudien vorgenommen. Neben den einbezogenen Studien eines bereits existierenden Reviews von Dorfner et al. (2017) wurden aktuelle Arbeiten zwischen 2016-2019 ergänzt. Dazu wurden die Datenbanken (peDOCS, ERIC, PsychINFO, Taylor \& Francis Online, Wiley Online Library, Springer Link) und Suchbegriffe (z. B. für PeDOCS: Videostudie, Videoanalyse, Unterrichtsbeobachtung) des Reviews von Dorfner et al. (2017) verwendet. Inklusive der bei Dorfner et al. (2017) einbezogenen Publikationen wurden 95 empirische Publikationen analysiert (vgl. Heinitz \& Nehring in diesem Thementeil).

Auf Basis der recherchierten Forschungsarbeiten wurden zentrale (Sub-)Dimensionen in den drei Fachdidaktiken herausgearbeitet. In den Fächern Sport und Geschichte wurde hierfür der Ansatz des narrativen Reviews gewählt, in den Naturwissenschaften wurde aufgrund der hohen Anzahl einbezogener Publikationen eine tabellarische Liste aller genannten Qualitätsaspekte erstellt.

Darauf aufbauend erfolgte in den Einzelbeiträgen ein Abgleich der identifizierten Dimensionen, Subdimensionen und Indikatoren von Unterrichtsqualität mit dem Syntheseframework. Dabei wurde überprüft (siehe Abschn. 3.1), inwiefern in den 
untersuchten Fächern bzw. Fächergruppen Überschneidungen zwischen dem Framework und den fachspezifischen Beschreibungen von Unterrichtsqualität identifiziert werden können (Fragestellung 1) und welche (Sub-)Dimensionen in den drei Fachdidaktiken nicht thematisiert wurden (Fragestellung 2). Aufgrund der in den Fächern Sport und Geschichte überschaubaren Publikationslage wurde von den Autoren ein interpretierender Abgleich des Faches mit dem Syntheseframework vorgenommen. Um die große Anzahl an entsprechenden Publikationen in den Naturwissenschaften handhabbar zu machen, wurde die Übereinstimmung zwischen fachdidaktischen Aspekten und Syntheseframework dort mittels einer dreistufigen Skala überprüft (,,vollständige Übereinstimmung“, „teilweise Übereinstimmung“ und „starke Abweichung"). Für den vorliegenden Beitrag wurden die obigen Zuordnungen der fachdidaktischen Aspekte zum Syntheseframework anschließend im Gesamtteam des vorliegenden Beitrags diskutiert und entsprechende Revisionen vorgenommen, um ein geteiltes Verständnis der (Sub-)Dimensionen - und somit eine Vergleichbarkeit der jeweiligen fachspezifischen Einordnungen - sicherzustellen. Abschließend erfolgte eine Auseinandersetzung mit der Frage, welche fachspezifischen Anpassungen und Ergänzungen des Syntheseframeworks notwendig sind, um die jeweiligen fachspezifischen Besonderheiten abbilden zu können (Fragestellung 3; siehe Abschn. 3.2). Die Unterscheidung, ob es sich um eine notwendige fachspezifische Ergänzung oder aber lediglich um eine fachspezifische Anpassung handelt, wurde aufgrund der Konzeption der (Sub-)Dimensionen des Syntheseframeworks getroffen (siehe Praetorius und Charalambous 2018; siehe auch Praetorius et al. in diesem Thementeil). Dissense wurden im Gesamtteam diskutiert und in konsensuale Einschätzungen überführt.

\section{Ergebnisse}

\subsection{Abgedeckte (Sub-)Dimensionen des Syntheseframeworks in den fachspezifischen Diskursen}

Für die erste Fragestellung - Überschneidungen des Syntheseframeworks mit fachspezifischen Ansätzen - und die zweite Fragestellung - im Syntheseframework fehlende fachdidaktische Aspekte - zeigte sich folgendes Bild für die einzelnen Fächer/ Fächergruppen:

Für die naturwissenschaftlichen Fächer ließ sich eine sehr hohe Passung des fachspezifischen Diskurses zum Syntheseframework feststellen. Alle sieben Dimensionen des Syntheseframeworks konnten in den fachspezifischen Publikationen identifiziert werden (siehe Tab. 1). Dies galt größtenteils auch für die Subdimensionen: Lediglich zwei der im Syntheseframework enthaltenen 21 Subdimensionen fanden sich in naturwissenschaftsdidaktischen Publikationen nicht explizit (konstruktiver 
Umgang mit Fehlern und Schwierigkeiten von Schüler*innen beim Üben sowie Beziehung der Schüler*innen untereinander). ${ }^{4}$

Obwohl das Fach Sport mit seinen Zielen, Inhalten und Methoden deutliche Unterschiede zum Fach Mathematik aufweist, fand sich eine vergleichsweise hohe Passung zum Syntheseframework: Fünfzehn der 21 Subdimensionen des Syntheseframeworks wurden auch in der Unterrichtsforschung des Faches Sport explizit diskutiert (siehe Tab. 1). Die Subdimensionen der Dimensionen Klassenführung und sozio-emotionale Unterstützung wurden dabei vollständig abgedeckt. Bei den Dimensionen Auswahl und Thematisierung von Inhalten und Fachmethoden (2 von 4 Subdimensionen), kognitive Aktivierung (in der Konzeptualisierung als motorischkognitive Aktivierung mit 3 von 4 Subdimensionen) und formatives Assessment (1 von 4 Subdimensionen) wurden lediglich Teilaspekte im Fach Sport berücksichtigt.

Der fachspezifische Forschungsstand im Fach Geschichte wies im Hinblick auf drei der sieben Dimensionen (Auswahl und Thematisierung von Inhalten und Fachmethoden, kognitive Aktivierung und Unterstützung des Übens) eine Entsprechung zum Syntheseframework auf (siehe Tab. 1). In Bezug auf diese Dimensionen fand sich auch für die Subdimensionen eine hohe Überschneidung mit dem Syntheseframework (Auswahl und Thematisierung von Inhalten und Fachmethoden: 3 von 4 Subdimensionen; kognitive Aktivierung: 4 von 4 Subdimensionen; Unterstützung des Übens: 1 von 2 Subdimensionen). Die Dimensionen Klassenführung, sozio-emotionale Unterstützung, Unterstützung des Lernens aller Schülerinnen und Schüler und formatives Assessment wurden allerdings bislang im fachspezifischen Diskurs nicht oder so gut wie nicht berücksichtigt.

Zusammenfassend lässt sich feststellen, dass eine große Überschneidung der fachspezifischen Forschungsstände zu Unterrichtsqualität mit den Dimensionen sowie Subdimensionen des Syntheseframeworks vorliegt (Fragestellung 1). Zudem wurde sichtbar, welche Dimensionen und Subdimensionen in den einzelnen Fächern bislang keine Rolle spielen (Fragestellung 2): Am deutlichsten ist dies für das Fach Geschichte zu erkennen, in dem einige Dimensionen des Syntheseframeworks in der bisherigen Literatur nicht aufgegriffen wurden. Im Fach Sport wurden insbesondere solche Dimensionen des Syntheseframeworks nur in Teilen thematisiert, die sich auf einzelne Schritte der prototypischen Lehr-Lernsequenz beziehen (Auswahl und Thematisierung von Inhalten und Fachmethoden, kognitive Aktivierung und formatives Assessment). In den naturwissenschaftlichen Fächern hingegen existieren nur vereinzelt Aspekte, die bislang im fachspezifischen Diskurs nicht explizit aufgegriffen wurden (in den Dimensionen Unterstützung des Übens und sozio-emotionale Unterstützung).

\footnotetext{
${ }^{4}$ Es kann jedoch diskutiert werden, ob sich diese beiden Subdimensionen implizit wiederfinden. So kann beispielsweise die Beziehung der Schüler*innen untereinander als Teilaspekt des Klassenklimas betrachtet werden, auch wenn die Beziehung der Schülerinnen untereinander nicht explizit fokussiert wird.
} 


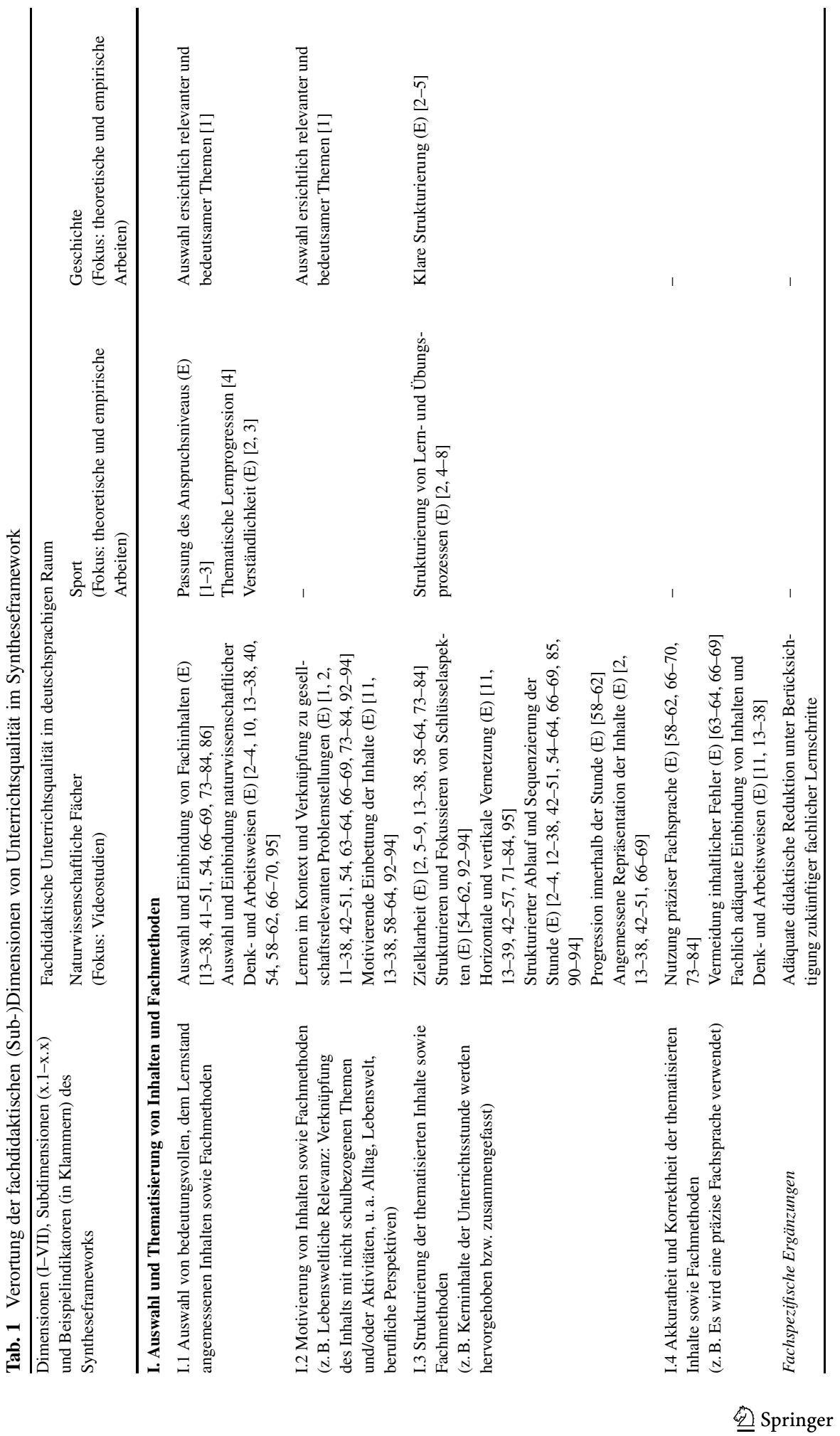




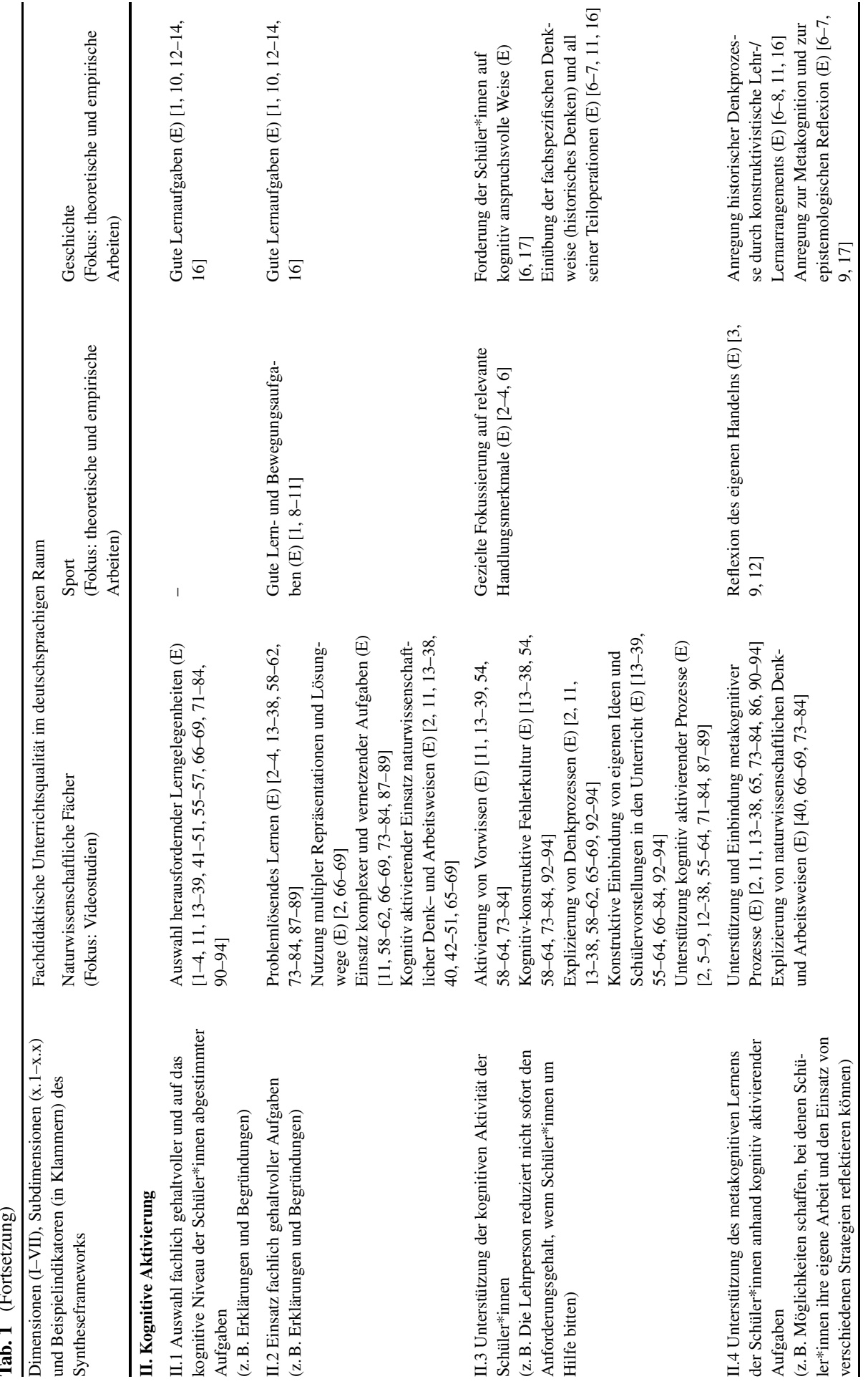




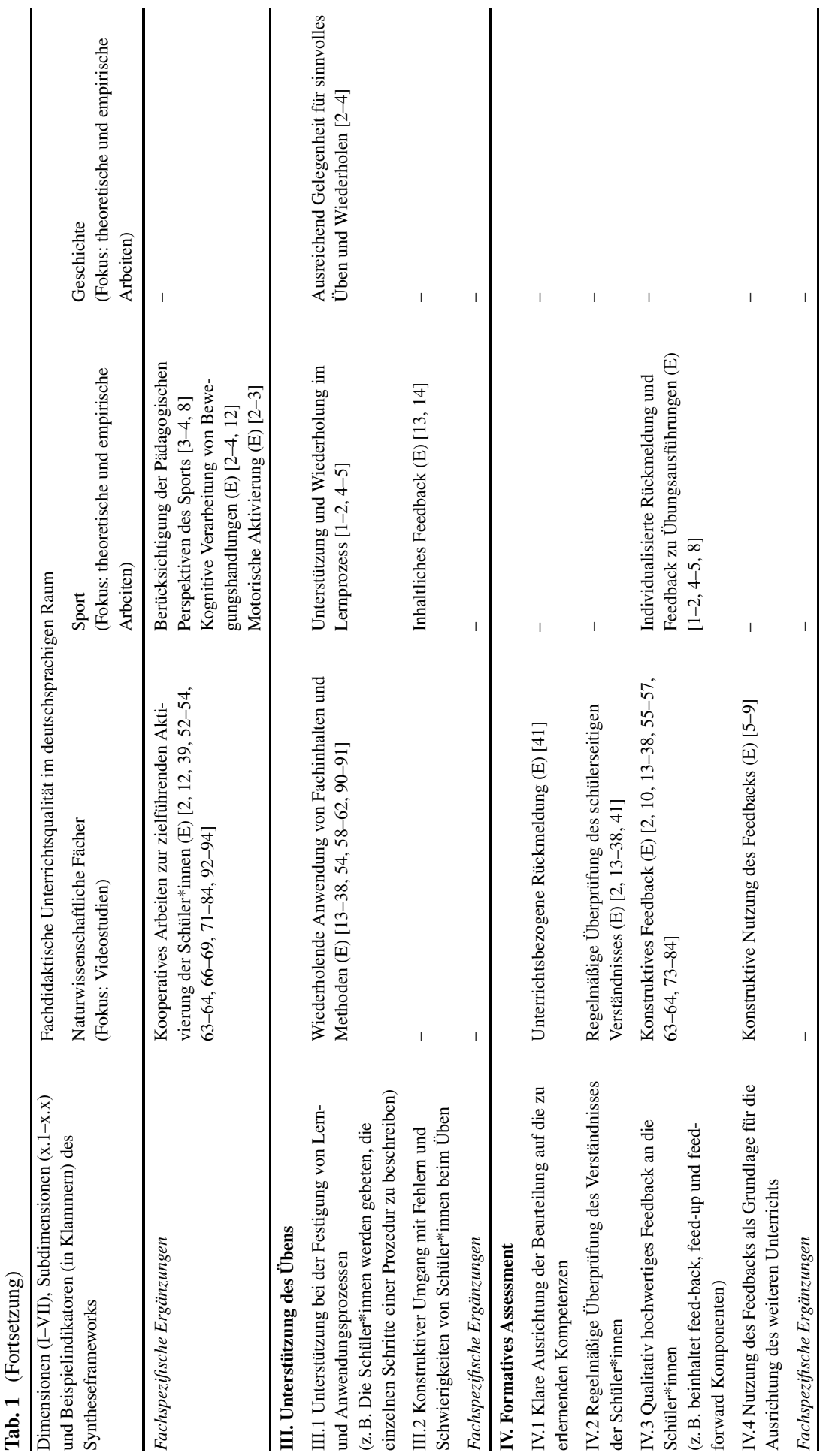




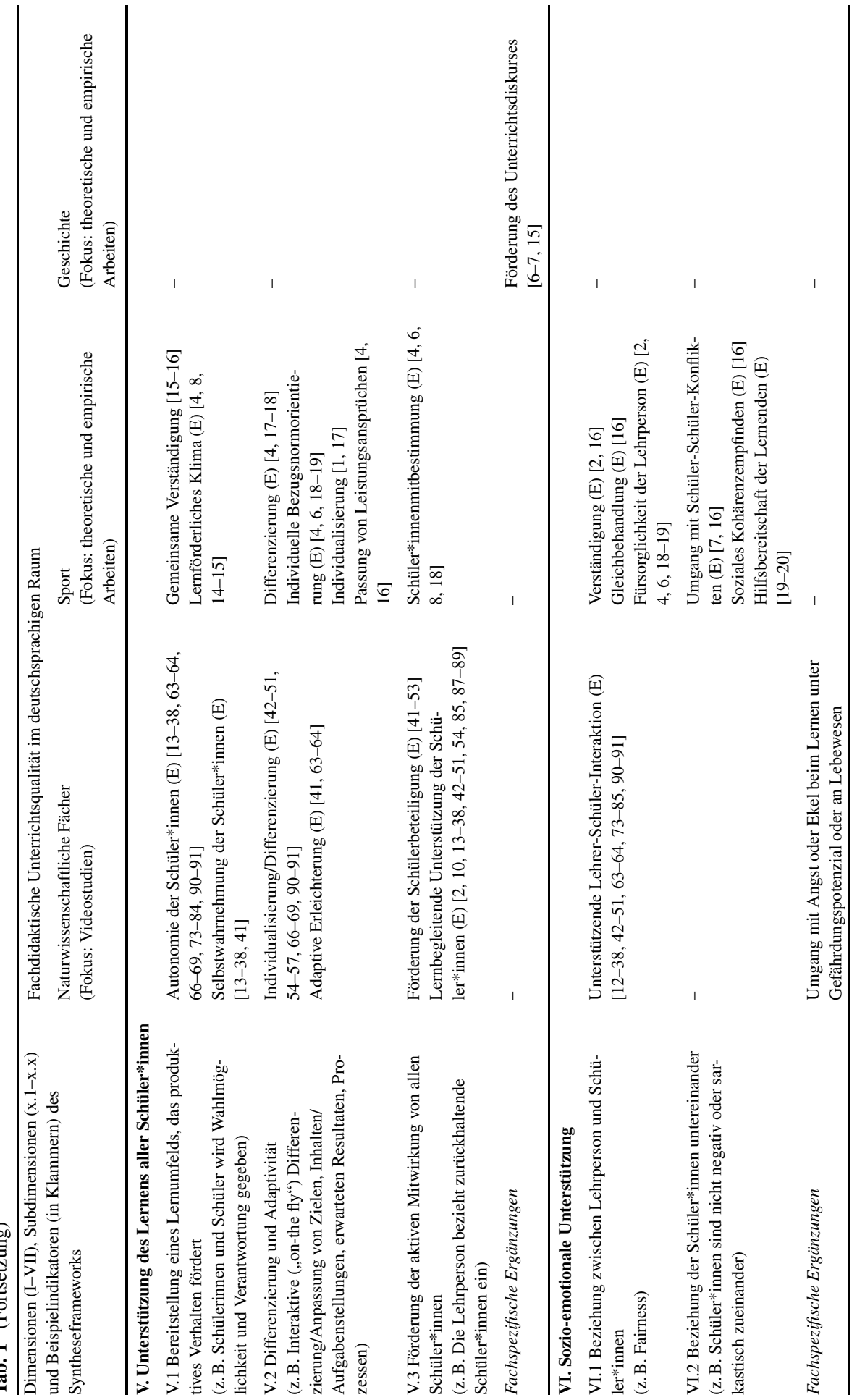




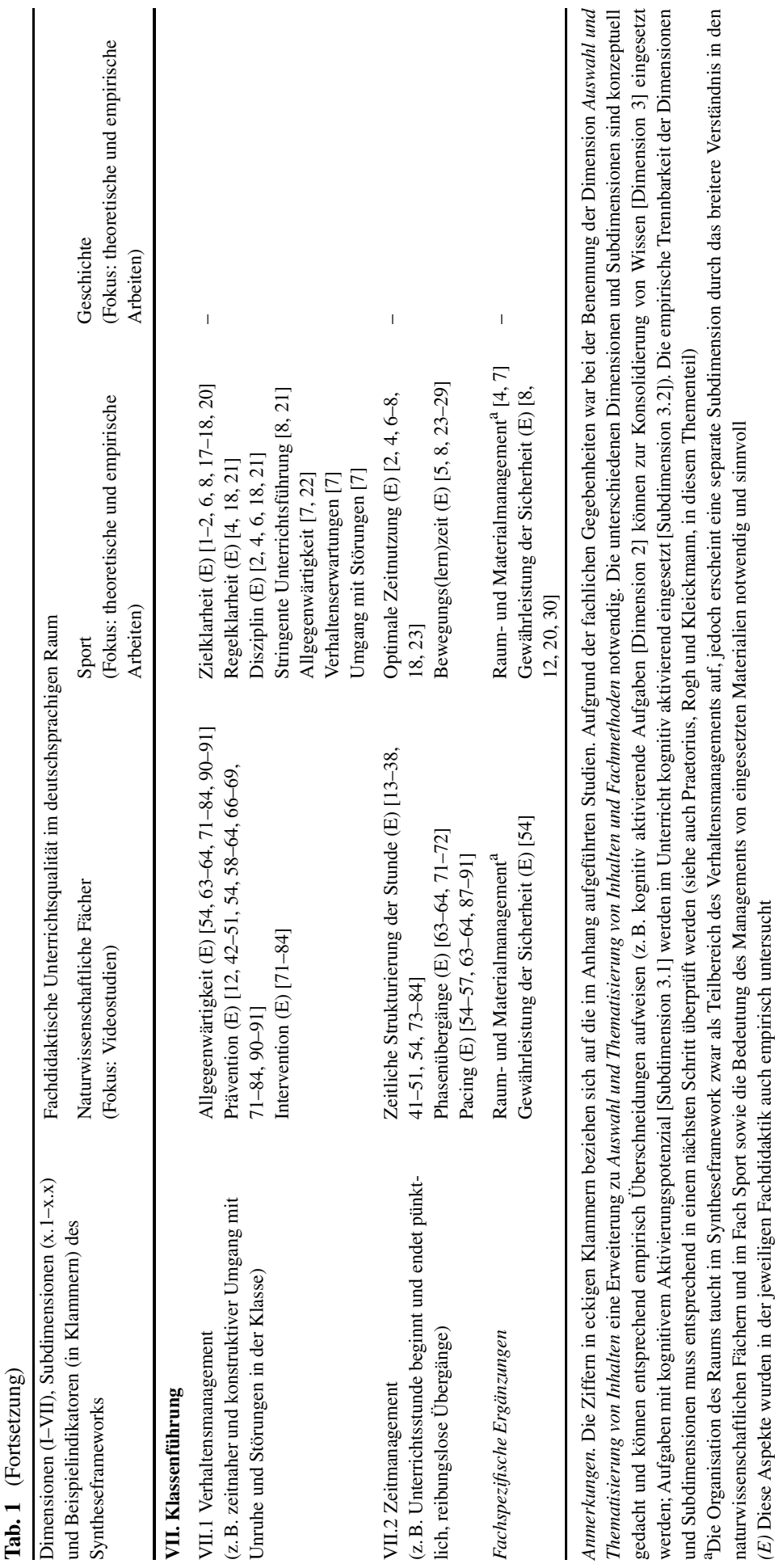




\subsection{Notwendige fachspezifische Anpassungen und Ergänzungen des Syntheseframeworks}

Auch wenn viele der unterschiedenen Dimensionen und Subdimensionen des Syntheseframeworks mit den Fachdiskursen übereinstimmen, bedeutet dies im Umkehrschluss nicht, dass mit diesen die Fachspezifität von Unterrichtsqualität vollständig abgebildet werden kann (Fragestellung 3). Dies wird aus den fachspezifischen Ergänzungen deutlich (siehe Tab. 1). Zwar wurde durch die Aufarbeitung des jeweiligen Forschungsstandes in den Fächern keine Ergänzung einer weiteren Dimension als notwendig erachtet, wohl aber einige Ergänzungen auf Ebene der Subdimensionen. Auf Basis des aktuellen Forschungsstandes wurden für drei der Dimensionen in jeweils einem der Fächer/Fächergruppen Ergänzungen als bedeutsam gesehen (Naturwissenschaften: Auswahl und Thematisierung von Inhalten und Fachmethoden, sozio-emotionale Unterstützung; Geschichte: Unterstützung des Lernens aller Schüler*innen). Für die Dimensionen Klassenführung (Naturwissenschaften; Sport) und kognitive Aktivierung (Naturwissenschaften; Sport) wurden zusätzliche Subdimensionen von zwei Fächern/Fächergruppen ergänzt.

Im Falle der naturwissenschaftlichen Fächer handelte es sich bei den Ergänzungen erstens um fachspezifische Formen der sozio-emotionalen Unterstützung bezüglich der Auseinandersetzung mit Inhalten: Eine besondere Rolle in diesen Fächern spielen die Antizipation von Angst, Respekt oder Ekel beim Umgang mit Lebewesen oder Gefahrstoffen. Des Weiteren kommt im Hinblick auf die Dimension Klassenführung aufgrund der Spezifik experimenteller Tätigkeiten der Gewährleistung der Sicherheit sowie dem Raum- und Materialmanagement hohe Bedeutung zu, da zum einen das Gefährdungspotential bei der Verwendung von Geräten und Chemikalien beachtet werden muss, aber auch die Besonderheiten des Fachraumes speziell in Experimentierphasen, sowohl mit Blick auf die Sicherheit als auch auf die vorhandene Ausstattung. Besondere Aufmerksamkeit im Bereich der kognitiven Aktivierung wurde in den konsultierten Studien auf kooperatives Arbeiten zur zielführenden Aktivierung der Schüler*innen gelegt, das in praktischen Arbeitsphasen, aber auch in der weiterführenden Diskussion der Lerninhalte eine wichtige Rolle einnimmt wenngleich diese Ergänzung aus Perspektive der allgemeinen Unterrichtsforschung sowie der anderen Fächer nicht spezifisch für die naturwissenschaftlichen Fächer erscheint. Angesichts der Besonderheiten naturwissenschaftlicher Curricula, die hierarchisch und aufeinander aufbauend konstruiert sein können (,Spiralcurricula“), ist bei der Dimension Auswahl und Thematisierung von Inhalten und Fachmethoden auf eine adäquate didaktische Reduktion unter Berücksichtigung zukünftiger fachlicher Lernschritte zu achten: Fachinhalte sollten für den Lernstand der Schülerinnen und Schüler angemessen sein, jedoch keine inhaltliche Reduktion erfordern, die später zu Konflikten im weiteren Aufbau von fachlichen Vorstellungen führt und ein Umlernen nach sich zieht („Prinzip der Ausbaufähigkeit“, Risch und Peifer 2018).

Ein Unterrichtsziel des Faches Sport ist es, den Schülerinnen und Schülern zu vermitteln, unter welchen Motiven und Sinnperspektiven sie Bewegung und Sport betreiben können (u. a. Sport treiben, um gesund zu bleiben; um etwas zu leisten; um Gemeinschaft zu erleben; Neumann und Balz 2013). Die Pädagogischen Perspektiven des Sportunterrichts zu berücksichtigen und die Schülerinnen und Schü- 
ler zur kognitiven Verarbeitung von Bewegungshandlungen anzuregen, sind zentrale Aspekte der kognitiven Aktivierung im Sportunterricht. Weiterhin schließen Lehrund Lernprozesse im Fach Sport unmittelbar an Bewegungshandlungen an. Damit läuft die kognitive Auseinandersetzung mit dem Lerngegenstand in einem ständigen Wechsel von kognitiver und motorischer Aktivität ab (Niederkofler und Amesberger 2016). Entsprechend erscheint aus sportdidaktischer Sicht auch die motorische Aktivierung eine bedeutsame Ergänzung der kognitiven Aktivierung zu sein. Für die Dimension Klassenführung stellt sich für das Fach Sport die Besonderheit, dass eine hohe Verletzungsgefahr besteht und die Gewährleistung der Sicherheit oberste Priorität hat. Dabei ergeben sich besondere Anforderungen an das Material- und Raummanagement. Neben der Vielzahl an Sportgeräten und -materialien müssen auch die Position und die Bewegungen der Schülerinnen und Schüler permanent organisiert werden, weil der Sportplatz keine feste Raumordnung (bspw. Sitzreihen) aufweist und sich die Schülerinnen und Schüler im Raum frei bewegen.

Aus Sicht der geschichtsdidaktischen Unterrichtsforschung müsste das Syntheseframework stärker die Qualität der unterrichtlichen Kommunikation in den Blick nehmen. Unterrichtstheoretisch wird in der Geschichtsdidaktik hervorgehoben, dass die im Unterricht angestrebte Elaboration historischer Kompetenzen durch die Kommunikation der beteiligten Akteure geschehen muss (Bracke et al. 2018). Kultivierte Unterrichtsdiskurse sind entsprechend eine wichtige Voraussetzung für erfolgreiche Lernprozesse im Geschichtsunterricht. Empirisch scheint sich diese Einsicht zu bestätigen (vgl. Cohors-Fresenborg et al. 2014; Nowińska 2016). Eine Ergänzung ist erforderlich, da weder die drei Basisdimensionen von Unterrichtsqualität (Klieme et al. 2001) noch das Syntheseframework eine entsprechende (Sub-)Dimension der Diskursqualität explizit berücksichtigt haben.

Nicht aus Tab. 1 ersichtlich, aber den Einzelbeiträgen zu entnehmen, ist eine weitere Ebene der Fachspezifität, die sich in den Analysen als besonders bedeutsam herausgestellt hat: die Ebene der konkreten Indikatoren der Subdimensionen, also derjenigen Verhaltensaspekte, die in konkreten Unterrichtssituationen beobachtbar sind und eine höhere oder niedrigere Qualität indizieren. Dies soll exemplarisch für jedes Fach/jede Fächergruppe in Bezug auf die Dimension kognitive Aktivierung erläutert werden.

Für die naturwissenschaftlichen Fächer stellt die Einbindung von Schülervorstellungen in den Unterricht ein zentrales Thema im Rahmen der kognitiven Aktivierung dar. Der Gegenstand naturwissenschaftlicher Untersuchungen, z. B. im Chemieunterricht, erstreckt sich von einer unmittelbar sichtbaren makroskopischen Ebene bis hin zu einer submikroskopischen Ebene, mit einer zusätzlichen Nutzung der Symbolebene, um den Untersuchungsgegenstand näher zu beschreiben. Wie in naturwissenschaftsdidaktischen Forschungen wiederkehrend gezeigt, beginnen Schülerinnen und Schüler naturwissenschaftliches Lernen mit bestehenden anschlussfähigen Vorstellungen oder aber fachlich nicht tragfähigen Vorstellungen über Naturphänomene, die den Ausgangspunkt für Lernprozesse darstellen. Qualitätsvoller naturwissenschaftlicher Unterricht aktiviert, integriert und/oder entwickelt diese bestehenden Vorstellungen, um eine anschlussfähige Entwicklung hin zu fachlich adäquaten Vorstellungen zu ermöglichen. Indikatoren für kognitive Aktivierung im naturwissenschaftlichen Unterricht fokussieren deshalb häufig auf die Möglichkeit, dass Schü- 
lerinnen und Schüler eigene Ideen und Vorstellungen äußern und die Lehrpersonen diese aufgreifen und konstruktiv einbinden.

Im Fach Sport schließt kognitive Aktivierung unmittelbar an Bewegungshandlungen an. Zur Steuerung dieser Bewegungshandlungen werden neben Wissensrepräsentationen auch motorische Repräsentationen benötigt. Motorische Repräsentationen beinhalten implizite kognitive Aspekte der Bewegungsausführung und sind im Gegensatz zu den expliziten Wissensrepräsentationen nur schwer verbalisierbar. Indikatoren kognitiver Aktivierung im Sportunterricht beinhalten daher neben der Anregung kognitiver Aktivität auch Maßnahmen der Lehrperson, um motorische Performanzen, d.h. das konkrete Bewegungshandeln im Sport, anzuregen. Längsschnittliche Befunde zu den Wirkungen einer solchen kognitiv-motorischen Aktivierung stehen bislang jedoch aus.

Für das Fach Geschichte wären für die Dimension der kognitiven Aktivierung die zentralen Operationen historischen Denkens zu nennen. Im Fach Geschichte sind auf der Ebene der Indikatoren zur Identifikation einzelner für die Dimension der kognitiven Aktivierung relevanter tiefenverarbeitender Lernprozesse Spezifikationen erforderlich, die hier nur exemplarisch angeführt werden können. Historische Sachverhaltsanalysen rekonstruieren beispielsweise einen vergangenen Sachverhalt auf der Basis einer prinzipiell lückenhaften und in sich disparaten Quellenlage. Historische Sachurteilsbildungen dagegen konstruieren kausale Beziehungen zwischen historischen Sachverhalten, wobei im Kern immer theoretische oder theorieförmige Annahmen stehen müssen. Entsprechend dem aktuellen Verständnis von adäquater Vermittlung in der Geschichtsdidaktik wird davon ausgegangen, dass diese Denkleistungen zudem in einem narrativen und moderat konstruktivistischen Rahmen angebahnt werden sollten - wenngleich sich die empirische Befundlage zu positiven Auswirkungen auf historische Denkleistungen noch ausbaufähig darstellt. Mit narrativ ist gemeint, dass historische Denkleistungen in erzählerischer Form prinzipiell auf gegenwärtige Orientierungsbedürfnisse ausgerichtet sein sollen; mit moderat konstruktivistisch ist ein epistemologischer Vorbehalt gemeint, mit dem zwischen Aussagen über historische Sachverhalte und den Sachverhalten selbst zu unterscheiden ist. Von kognitiver Aktivierung wäre also zu sprechen, wenn historische Denkleistungen in diesem Sinne angebahnt würden.

\section{Diskussion}

Das Anliegen des vorliegenden Beitrags bestand darin, fachspezifische und generische (Sub-)Dimensionen von Unterrichtsqualität einer vergleichenden Analyse zu unterziehen. Dieser Vergleich zeigte zunächst, dass sich auf einer allgemein formulierten Ebene große Überschneidungen in den Diskursen zur Unterrichtsqualität zwischen der generisch ausgerichteten sowie mathematikdidaktischen Unterrichtsforschung und denjenigen der hier berücksichtigten Fachdidaktiken feststellen lassen. Dies deutet darauf hin, dass es über Fächergrenzen hinweg eine bemerkenswerte Schnittmenge zentraler Dimensionen und Subdimensionen von Unterrichtsqualität zu geben scheint. Auf Ebene der übergreifenden Dimensionen scheint Unterrichts- 
qualität somit zumindest über die Fächer Mathematik, Naturwissenschaften, Sport und Geschichte generisch beschrieben werden zu können.

Zugleich zeigt die vergleichende Analyse jedoch, dass der bisherige Fokus auf den Mathematikunterricht in der allgemeinen empirischen Unterrichtsforschung dazu geführt hat, dass einerseits Anpassungen der existierenden Dimensionen, Subdimensionen sowie Indikatoren notwendig sind und andererseits Ergänzungen weiterer Subdimensionen und Indikatoren, die in den betreffenden Fächern als relevant angesehen werden, in Erwägung gezogen werden sollten. Solche Ergänzungen - wie beispielsweise die Diskursqualität - wiederum könnten auch stimulierend für andere Fächer wirken, wie sich an den obigen Erläuterungen erkennen lässt.

\subsection{Fachspezifische Anpassungen und Ergänzungen}

Wenngleich ein Großteil der Dimensionen und Subdimensionen in allen untersuchten Fächern wiederzufinden war, wird in den Einzelbeiträgen deutlich, dass fachspezifische Anpassungen der (Sub-)Dimensionen sowie der zugehörigen Indikatoren notwendig sind. Diese erscheinen insbesondere auf Ebene der Indikatoren notwendig, da dort der direkte Bezug zum konkreten Gegenstand hergestellt wird. So hat beispielsweise die Dimension sozio-emotionale Unterstützung mit ihrer Subdimension Beziehung zwischen Lehrperson und Schüler*innen eine fachübergreifende Relevanz. Dagegen müssen die (operationalisierbaren) Indikatoren, welche dazu dienen, konkrete Unterrichtssituationen zu beurteilen, auf den Unterrichtsgegenstand bezogen sein. Im Fach Sport muss die Lehrperson beispielsweise dafür Sorge tragen, dass Schülerinnen und Schüler nicht gezwungen sind, sich körperlich zu exponieren (z. B. Vorturnen vor der Klasse), um Beschämung zu reduzieren und einen vertrauensvollen Umgang zwischen Lehrperson sowie Schülerinnen und Schülern herzustellen. In anderen Fächern fällt diese körperliche Exponiertheit weniger ins Gewicht und andere Indikatoren rücken stärker in den Fokus (z. B. emotionale Unterstützung bei Misserfolgserfahrungen).

Über die Ebene der Indikatoren hinaus wurden zudem für alle hier berücksichtigten Fächer jeweils fachspezifische Subdimensionen identifiziert und konzeptualisiert, die in dieser Form in der generisch ausgerichteten sowie mathematikdidaktischen Unterrichtsforschung aktuell nicht diskutiert werden. Entsprechend sollten diesbezügliche Ergänzungen in Betracht gezogen werden - wenngleich in diesem Zusammenhang eine zentrale Herausforderung darin bestehen wird, die Anzahl an Dimensionen und Subdimensionen so zu begrenzen, dass sie in empirischen Untersuchungen umsetzbar sind.

Nicht systematisch berücksichtigt haben wir in unseren bisherigen Analysen zudem, ob die identifizierte Fachspezifität darauf zurückzuführen ist, dass die jeweiligen Subdimensionen nur in einem Fach relevant sind (z. B. Fokus auf Bewegungshandlungen und deren entsprechende Begleitung im Fach Sport) oder aber die zusätzlich identifizierten Aspekte auch potenziell relevant für andere Fächer sind und bislang lediglich in diesen nicht explizit diskutiert wurden (z. B. Förderung des Unterrichtsdiskurses im Fach Geschichte). Neben den jeweils nur für ein Fach genannten Ergänzungen konnten auch fachspezifische Ergänzungen identifiziert werden, die nicht alle, aber mehrere Fächer betreffen (z. B. Gewährleistung der Sicherheit in 
den Naturwissenschaften sowie im Sport). Die Überschneidungen sprechen dafür, dass diese Aspekte auch in der allgemeinen Unterrichtsforschung stärker thematisiert werden sollten. Offen ist hierbei, inwiefern es sich um Subdimensionen handelt, die wie die sieben unterschiedenen Dimensionen prinzipiell für alle Fächer Relevanz haben oder ob es bestimmte Fächergruppen gibt, die sich durch bestimmte Ergänzungen charakterisieren. So spielen Sicherheitsaspekte vermutlich vor allem in jenen Fächern eine Rolle, in denen Gefahren für die Physis nicht unwahrscheinlich sind. Das in den naturwissenschaftlichen Fächern und im Fach Sport ergänzte Raum- und Materialmanagement hingegen könnte man vermutlich in einigen weiteren Fächern als zu berücksichtigenden Aspekt hinzunehmen, wenngleich es je nach Fach eine unterschiedliche Relevanz haben dürfte. Eine weitere Herausforderung liegt darin, dass die einzelnen Fächer den (Sub-)Dimensionen in ihrer konzeptuellen Auseinandersetzung sowie in der empirischen Erforschung bislang eine unterschiedliche Bedeutsamkeit zuweisen. Die Dimension Klassenführung spielt beispielsweise im Fach Sport wegen der räumlichen Gegebenheiten sowie der potenziellen Verletzungsgefahr eine zentrale Rolle. Entsprechend existiert hierzu mittlerweile eine Reihe an fachspezifischen Publikationen. In der geschichtsdidaktischen Unterrichtsforschung hingegen spielt die Dimension Klassenführung bislang eine untergeordnete Rolle, möglicherweise weil das Fach auf eine differenzierte eigene Theoriebildung zurückblickt, die stark auf hermeneutischen Traditionen fußt.

\subsection{Implikationen aus den Anpassungen und Ergänzungen}

Die Implikationen der oben angesprochenen Anpassungen und Ergänzungen konnten im Rahmen der vorliegenden Synthese nur angedacht werden und müssen zukünftig weiter diskutiert werden. So ist bislang offen, inwiefern man bei z.T. sehr unterschiedlichen Indikatoren bestimmter (Sub-)Dimensionen letztlich noch von demselben Konstrukt sprechen kann. Dies betrifft selbst Dimensionen wie diejenige der Klassenführung, von denen bislang in der allgemeinen empirischen Unterrichtsforschung davon ausgegangen wurde, dass es sich um generische Unterrichtsdimensionen handelt. Um dieser Frage weiter nachgehen zu können, müsste zukünftig diskutiert werden, wodurch sich das Ausmaß an Fachspezifität von Unterrichtsaspekten überhaupt bestimmen lässt. Dies wird in bisherigen Publikationen kaum expliziert. Martin-Raugh et al. (2016) argumentierten, dass die Frage nach der Fachspezifität weniger von der konkreten Operationalisierung abhängt, als eher davon, ob die Bedeutung des zugrundeliegenden Konstrukts über Fächer hinweg dieselbe ist. Um eine entsprechende Einschätzung zu ermöglichen, sind jedoch detaillierte und theoriebasierte Konstruktbeschreibungen notwendig, die bislang kaum vorliegen. In ihrem Übersichtsbeitrag zeigten Praetorius und Charalambous (2018), dass manche Unterrichtsdimensionen je nach Forschungstradition als generisch oder als fachspezifisch interpretiert werden (z. B. kognitive Aktivierung). Die von Praetorius und Charalambous (2018) vorgeschlagene Systematik von rein generischen, über kombiniert fachspezifisch-generische bis hin zu rein fachspezifischen (Sub-)Dimensionen von Unterrichtsqualität lässt sich allerdings mit den vorliegenden Analysen nicht unterstützen, da selbst Dimensionen wie Klassenführung ein bedeutsames Ausmaß an Fachspezifität aufweisen. Unseren Analysen zufolge hängt die Fachspezifität 
vielmehr von der fokussierten Ebene ab (Dimension, Subdimension oder Indikator) und lässt sich insbesondere auf Ebene der Indikatoren ansiedeln.

Auf Dimensionsebene deutet sich an, dass die sieben im Syntheseframework unterschiedenen Dimensionen prinzipiell in allen Fächer bedeutsam sein könnten. Fraglich ist lediglich, ob die notwendigen Anpassungen und Ergänzungen auf Indikatoren- und Subdimensionsebene dazu führen, dass sich das dahinterstehende Konstrukt (z. B. Klassenführung) auf Dimensionsebene verändert. Würden die Dimensionen in den Fächern unterschiedliche Konstrukte abbilden, wäre es zumindest notwendig, das jeweilige Fach in der Benennung der Dimension zu ergänzen (z. B. Klassenführung im Sport).

Dass solche Überlegungen nicht nur spezifisch für den Gegenstand Unterricht relevant sind, zeigen Auseinandersetzungen in der interkulturellen Forschung, in der das Problem der Äquivalenz ebenfalls auf mehreren Ebenen diskutiert wird (z.B. Rippl und Seipel 2008; Bachleitner et al. 2014). Dabei werden in der Regel Konstruktäquivalenz und Messäquivalenz mit entsprechenden Unterkategorien unterschieden. So wird unter Konstruktäquivalenz verstanden, dass unter dem theoretischen Konstrukt tatsächlich in allen Kulturen (hier: Fächern) das Gleiche verstanden wird; d. h. im vorliegenden Fall verstehen alle Fächer Unterrichtsqualität als ein multidimensionales, auf die Entwicklung von Schülermerkmalen (z. B. Leistungsentwicklung) ausgerichtetes Konstrukt. Offenbar lassen sich auch alle sieben Dimensionen des Syntheseframeworks in allen Fächern konzeptualisieren. Für bestimmte Dimensionen sind zwar Anpassungen erforderlich (z.B. kognitive und motorische Aktivierung im Sport), die grundsätzliche Terminologie kann jedoch beibehalten werden. Auf der Subdimensionsebene und insbesondere auf der Ebene der Indikatoren sind dann größere Anpassungen notwendig (z.B. Förderung des Unterrichtsdiskurses in Geschichte; Gewährleistung der Sicherheit in den naturwissenschaftlichen Fächern und im Fach Sport). In der interkulturellen Forschung würde man dies als nicht vollständige Konstruktäquivalenz aufgrund unzureichender kategorialer Äquivalenz bezeichnen. Fragen der Messäquivalenz, also der Vergleichbarkeit der empirischen Erfassungen, werden in der Regel erst bei der Datenanalyse in der Prüfung von Faktorstrukturen diskutiert (z.B. faktorielle, metrische oder skalare Äquivalenz beim Vergleich mehrerer Gruppen/Fächer). Beim Streben nach Konstrukt- und Messäquivalenz über Fächer hinweg besteht die Gefahr, dass letztendlich zwar vergleichbare Konstrukte untersucht werden, dass allerdings relevante Subdimensionen oder Indikatoren einzelner Fächer aus dem Blick geraten.

Wir würden entsprechend argumentieren, dass zukünftig verstärkt kritisch diskutiert werden sollte, ob das alleinige Priorisieren von fachübergreifender Äquivalenz und der entsprechende Fokus auf der Identifikation generischer Aspekte von Unterrichtsqualität, angemessen ist. Unserer Einschätzung nach könnte es - ähnlich unserem hier gewählten Vorgehen - zielführend sein, bewusst beides in den Blick zu nehmen: diejenigen Aspekte, die sich als äquivalent beschreiben lassen sowie diejenigen, die spezifisch für bestimmte Fächer sind. 


\subsection{Herausforderungen bei der Interpretation der Befunde}

Bei den Diskussionen im interdisziplinären Team wurden immer wieder Herausforderungen bezüglich einer eindeutigen vergleichenden Interpretation der aktuellen Befundlage zu Unterrichtsqualität in den Fachdidaktiken deutlich. Auf drei zentrale Punkte wird im Folgenden eingegangen.

Erstens wurde in der vorliegenden Arbeit eine Zusammenfassung unterschiedlichster Befunde vorgenommen, obgleich sich die Evidenz zu den einzelnen Dimensionen unterscheidet: So wurde in den Analysen deutlich, dass je nach Fach zu den Dimensionen und Subdimensionen unterschiedlich viele Arbeiten vorlagen sowie diese zum Teil vorrangig konzeptuell, zum Teil vorrangig empirisch ausfielen. Bei den empirischen Arbeiten wiederum handelte es sich nur zum Teil um experimentelle Studien sowie Längsschnittstudien, die Befunde zur Wirksamkeit der betreffenden Aspekte bieten. Entsprechend unterscheidet sich die belastbare Evidenz, die zur empirischen Bedeutsamkeit der jeweiligen Unterrichtsaspekte vorliegt. Dies gilt jedoch nicht nur für die Unterrichtsforschung in den Fachdidaktiken, sondern auch für die allgemeine Unterrichtsforschung (Helmke 2017; Seidel und Shavelson 2007).

Zweitens weisen vereinzelte Arbeiten darauf hin, dass sich die unterschiedenen Unterrichtsdimensionen in ihrer Funktion unterscheiden und entsprechend auch die Begründungen variieren, warum diese Dimensionen als Qualitätsaspekte von Unterricht gelten: Während einige Dimensionen direkt auf den Lernprozess der Schülerinnen und Schüler ausgerichtet sind (z.B. kognitive Aktivierung), sind andere Dimensionen so konzipiert, dass sie diesen nur indirekt beeinflussen (z. B. Klassenführung) (siehe Charalambous und Praetorius 2020; Openshaw und Clarke 1970). Entsprechend müsste in Zukunft in den einzelnen Fächern, aber auch in der allgemeinen Unterrichtsforschung, verstärkt die Funktion der einzelnen Dimensionen mitdiskutiert werden, wenn es um die Bedeutsamkeit von Aspekten der Unterrichtsqualität geht.

Drittens wurde in der fachübergreifenden Diskussion um bedeutsame Aspekte von Unterrichtsqualität rasch deutlich, dass Unterrichtsqualität nicht losgelöst von den Zielen, Inhalten und Methoden des Unterrichts betrachtet werden kann (siehe auch Neumann 2018): Die Qualität von Unterricht wird in der allgemeinen empirischen Unterrichtsforschung in Relation zur Erreichung gesetzter Lernziele auf Seiten der Schülerinnen und Schüler definiert. Darüber hinaus wird in zahlreichen Publikationen auf die Figur des didaktischen Dreiecks verwiesen (z. B. Reusser 2008): Unterricht konstituiert sich neben den handelnden Lehrpersonen sowie den Schülerinnen und Schülern in zentraler Weise durch den Unterrichtsgegenstand. Nimmt man beides zusammen, wird deutlich, dass eine adäquate Auseinandersetzung mit (Sub-)Dimensionen der Unterrichtsqualität eigentlich nur dann erfolgen kann, wenn diese im Kontext einer expliziten Thematisierung von zu erreichenden fachspezifischen Erziehungs- und Bildungszielen und der unterschiedlichen Lerngegenstände erfolgt. Wie in den Einzelbeiträgen ersichtlich, charakterisiert sich der jeweilige Gegenstand durch ein Zusammenspiel von Zielen, einbezogenen Inhalten sowie den spezifischen Methoden eines Fachs (siehe auch Abb. 1).

Die Abhängigkeit von Unterrichtsqualität von Zielen, Inhalten und Methoden stellt insbesondere für die generische empirische Unterrichtsforschung eine große 
Abb. 1 Bedeutung des Verhältnisses von Ziel, Inhalt, Methode und Unterricht für die Erforschung von Unterrichtsqualität

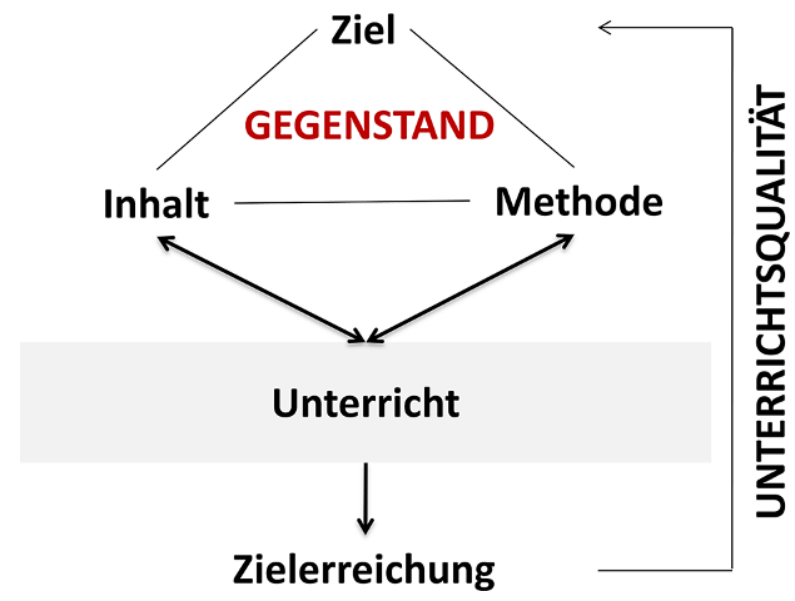

Herausforderung dar: Werden diese Aspekte bei der Erfassung von Unterrichtsqualität nicht berücksichtigt, kann nicht klar bestimmt werden, ob die Ausprägungen bestimmter Unterrichtsdimensionen ausschließlich Ausdruck einer hohen oder niedrigen Unterrichtsqualität sind oder ob sie auf eine unterschiedliche Ausrichtung des Unterrichts aufgrund von spezifischen Zielen, Inhalten und Methoden zurückzuführen sind. Erziehungswissenschaftlerinnen und -wissenschaftlern sowie Pädagogischen Psychologinnen und Psychologen fehlt für die explizite diesbezügliche Auseinandersetzung in der Regel die notwendige fachliche Expertise. Entsprechend erfordert die Berücksichtigung dieser Abhängigkeiten eine enge Zusammenarbeit der allgemeinen empirischen Unterrichtsforschung mit den jeweiligen Fachdidaktiken. Aber auch innerhalb der jeweiligen Fachdidaktik stellt diese Komplexität eine Herausforderung dar, da die zu erreichenden Ziele, die zu behandelnden zentralen Inhalte sowie die für ein Fach charakteristischen Methoden zum Teil national wie international kontrovers und unterschiedlich diskutiert und zudem von Lehrpersonen unterschiedlich ausgelegt werden. Ein auf Gesellschaftskritik und Emanzipation abzielender Geschichtsunterricht beispielsweise wird Wert auf Ideologiekritik und die Analyse gesellschaftlicher Strukturen legen, wohingegen sich ein wissenschaftspropädeutischer Unterricht eher auf die wissenschaftsförmige Einübung zentraler heuristischer und interpretativer Fachoperationen konzentrieren würde.

In diesem Zusammenhang erscheint es zudem lohnend zu diskutieren, welche Dimensionen von Unterrichtsqualität für alle zentralen Ziele, Inhalte und Methoden hohe Relevanz haben und welche lediglich oder vornehmlich für bestimmte Ziel-Inhalts-Methoden-Konfigurationen bedeutsam sind. Kognitive Aktivierung beispielsweise ist für die eigenständige Planung eines Experiments zur Überprüfung einer Hypothese in den Naturwissenschaften hoch relevant, während es für das auf das Routinen bildende Einüben ausgerichtete Wiederholen einer prozeduralen Tätigkeit (z. B. wiederholtes Pipettieren) ohne Bezug zu Prozessen naturwissenschaftlicher Erkenntnisgewinnung sinnvollerweise nicht als Qualitätsdimension herangezogen werden sollte. Die in den Naturwissenschaftsdidaktiken häufig bemühte Unterscheidung zwischen hands-on-Prozessen und minds-on-Prozessen verweist auf eine ähnliche 
Sichtweise (Bybee 2006). Klassenführung hingegen ist unabhängig von den gewählten Zielen, Inhalten und Methoden notwendig - wenngleich die konkret eingeschätzte Qualitätsausprägung sehr wohl von diesen abhängig sein könnte. So macht es für die Erfassung von Klassenführung, die aktuell oftmals über stark am Frontalunterricht orientierte Indikatoren abgedeckt wird, einen zentralen Unterschied, welchen methodischen Ansatz Lehrpersonen für ihren Unterricht wählen: Nach unserer Einschätzung weisen die Indikatoren eine höhere Passung auf, wenn die Lehrperson Frontalunterricht durchführt, um neue Inhalte zu vermitteln, als wenn sie offene Unterrichtsformen nutzt. Es erscheint daher sinnvoll, in zukünftigen Untersuchungen stärker zu thematisieren, inwiefern sich die Ausprägung der Dimensionen von Unterrichtsqualität systematisch danach unterscheiden, welche Ziele, Inhalte und Methoden im Fachunterricht verfolgt werden.

\subsection{Limitationen der vorliegenden Arbeit}

Für die vorliegende Arbeit wurde eine kontrastierende Auswahl von Fächern aus den drei Fächergruppen Naturwissenschaften, musisch-ästhetische Fächer und Gesellschaftswissenschaften vorgenommen. Daher ist durch den Fokus auf die ausgewählten Fächer eine abschließende Beantwortung der Fragestellungen nicht möglich: Die Einordnung der fachspezifischen Diskurse zu Unterrichtsqualität in den Diskurs der allgemeinen empirischen Unterrichtsqualitätsforschung sowie die Notwendigkeit der Ergänzung von (Sub-)Dimensionen des Syntheseframeworks könnte mit einer anderen Auswahl an Fächern trotz der Bandbreite der hier einbezogenen Fächer ggf. anders ausfallen.

Die Ausrichtung der Fragestellungen geht zudem mit der Einschränkung einher, dass lediglich Aspekte des unterrichtlichen Angebots in den Blick genommen wurden, während Aspekte der Nutzung dieses Angebots durch die Schülerinnen und Schüler in der vorliegenden Arbeit nicht behandelt wurden (für einen Überblick zu Angebots-Nutzungs-Modellen siehe Vieluf et al. 2020). Auch die in AngebotsNutzungs-Modellen unterschiedenen Rahmenbedingungen (z. B. familiärer Hintergrund; schulische Rahmenbedingungen) und weitere Faktoren (z.B. Merkmale der Lehrperson) wurden im vorliegenden Beitrag nicht berücksichtigt.

Aufgrund der hohen Überschneidung der Diskurse in den Fächern Biologie, Chemie und Physik wurden diese als Fächergruppe behandelt, während die Fächer Sport und Geschichte einzeln betrachtet wurden. Auch dadurch bedingt wurde in den einbezogenen Fächern ein unterschiedlicher methodischer Zugang bezüglich der Suchstrategie der Publikationen gewählt. So wurde in Sport und Geschichte generell nach Publikationen zu Unterrichtsqualität gesucht, in den Naturwissenschaften wurde hingegen aufgrund der hohen Anzahl an vorhandenen Studien eine Fokussierung auf Videostudien vorgenommen. Entsprechend kann kritisch diskutiert werden, inwiefern der in den Fächern dargestellte Forschungsstand hinreichend verglichen werden kann.

Als Kommunikations- und Verständnisgrundlage wurde in der vorliegenden Arbeit das von Praetorius und Charalambous (2018) vorgeschlagene Syntheseframework herangezogen, da dies beansprucht, unterschiedene Dimensionen und Subdimensionen der bisherigen allgemeinen und mathematikdidaktischen empirischen 
Unterrichtsforschung möglichst umfassend abzubilden. Diese Wahl hat deutliche Auswirkungen auf die vorgestellten Ergebnisse, da diese mit einer anderen Systematik ggf. anders aussähen. So sieht die Unterteilung des komplexen Metakonstrukts Unterrichtsqualität in besser fassbare Teilaspekte in verschiedenen Fächern unterschiedlich aus und ist oft stärker vom Gegenstand als von inhaltsübergreifenden Unterrichtsdimensionen ausgehend konzipiert. Beispielsweise werden in der naturwissenschaftlichen Unterrichtsforschung einzelne Methoden (z. B. Experimentieren, Schulz 2011) als Ausgangspunkt genommen und die Frage nach der Qualität spezifisch für diese Methoden untersucht.

Die Notwendigkeit einer gemeinsamen Kommunikations- und Verständnisgrundlage legte für die Ausrichtung der vorliegenden Arbeit einen generisch ausgerichteten Ansatz nahe. Dies bedeutet jedoch auch, dass sich die in einem Fach etablierten Begrifflichkeiten und Strukturierungen im vorliegenden Beitrag nicht immer wiederfinden und einige Spezifika der einzelnen Fachdidaktiken durch die vorgenommene Einordnung in das Syntheseframework nicht abgebildet werden können. Beispielsweise können naturwissenschaftliche Denk- und Arbeitsweisen (z. B. das Experimentieren) sowohl Inhalt als auch Methode oder Ziel einer Stunde sein und erstrecken sich über mehrere Dimensionen und Subdimensionen des Syntheseframeworks (siehe Heinitz \& Nehring in diesem Thementeil).

Eine große Herausforderung der vorliegenden Arbeit bestand darin, zwischen fachspezifischen Anpassungen und notwendigen Ergänzungen zu unterscheiden. Die entsprechende Einordnung wurde diskursiv ausgehandelt. Dabei wurde deutlich, dass eine klare Unterscheidung zwischen Anpassung und Ergänzung nicht in allen Fällen möglich ist, da eine Zuordnung zum Teil je nach Argumentation zu dem einen oder anderen möglich wäre. Insbesondere in der interdisziplinären Zusammenarbeit können unterschiedliche Sichtweisen bei der Einordnung von Unterrichtsaspekten auftreten, die nicht in allen Fällen einvernehmlich lösbar sind. Während beispielsweise von den Vertretern der Naturwissenschaften die Subdimension Adäquate didaktische Reduktion unter Berücksichtigung zukünftiger fachlicher Lernschritte innerhalb der Dimension Auswahl und Thematisierung von Inhalten und Fachmethoden als eine fachspezifische Erweiterung gesehen wurde, könnte sie auch als Bestandteil der Subdimension Auswahl von bedeutungsvollen, dem Lernstand angemessenen Inhalten sowie Fachmethoden interpretiert werden.

Eine weitere zentrale Einschränkung bezieht sich auf die Aufarbeitung des jeweiligen Forschungsstandes in den einzelnen Fächern. Da es sich bei Unterricht um ein sehr komplexes Geschehen handelt, existieren in jedem Fach vielfältige Forschungsbemühungen $\mathrm{zu}$ unterschiedlichen Teilaspekten und in verschiedenen Forschungstraditionen. Diese in einem Review zusammenzufassen, ist ausgesprochen herausfordernd und nur bis zu einem gewissen Grad objektivierbar. Entsprechend kann nicht sicher davon ausgegangen werden, dass alle relevanten Arbeiten in den jeweiligen Fächern zum Thema Unterrichtsqualität in den Reviews aufgearbeitet wurden, und auch die Einordnung in das Syntheseframework könnte durch verschiedene Forschende eines Faches unterschiedlich ausfallen. Die Identifikation von notwendigen Ergänzungen des Syntheseframeworks bezieht sich zudem auf bislang durchgeführte Studien in diesem Bereich. Die dabei untersuchten (Sub-)Dimensionen sind unter Umständen nicht in jedem Fall ausschließlich die für das jeweilige Fach besonders 
zentralen (Sub-)Dimensionen, sondern in Teilen auch die eher leicht zu erfassenden Aspekte (z. B. findet sich im Fach Sport bislang ein empirischer Fokus auf die beiden Dimensionen Klassenführung und sozio-emotionale Unterstützung, da unter anderem die Frage nach kognitiver Aktivierung aus den oben genannten Gründen für das Fach Sport mit einigen konzeptuellen Herausforderungen einhergeht).

Für den Vergleich zwischen den ausgewählten Fächern war zudem herausfordernd, dass sich die einzelnen Fachdidaktiken in ihrer forschungsmethodischen Ausrichtung unterscheiden. Entsprechend existiert unterschiedlich viel empirische Forschung zum Thema Unterrichtsqualität in den einzelnen Fächern. Auch die Erhebungsmethoden variieren zwischen den Fächern (z. B. vornehmlich videobasierte Forschung in den Naturwissenschaften vs. vornehmliche Erfassung von Schülerwahrnehmungen in Sport). Durch diese Unterschiede zwischen den Fächern wurde auch in den einzelnen Reviews der Beiträge in diesem Heft eine teilweise unterschiedliche Methodik gewählt, was einen Vergleich zwischen den Fächern erschwert.

\subsection{Fazit}

Im vorliegenden Beitrag wurde der aktuelle Stand der Forschung zu Unterrichtsqualität über mehrere Fächer hinweg verglichen. Die besondere Herausforderung lag darin, die Fachspezifität und damit die Besonderheiten der jeweiligen Fächer herauszustellen und diese dennoch einem generischen Rahmenkonzept zuzuordnen. Dabei ist es ein vornehmliches Anliegen der einzelnen Fachdidaktiken, Unterrichtsqualität möglichst fachspezifisch abzubilden, während die allgemeine Unterrichtsforschung versucht, in dieser Fachspezifik Gemeinsamkeiten zu finden, welche in übergreifenden Konstrukten abgebildet werden können. In welchem Umfang fachspezifische Aspekte berücksichtigt werden müssen und inwieweit diese wiederum zu generischen Aspekten zusammengefasst werden können, konnte im vorliegenden Beitrag nur in einem ersten Schritt beleuchtet werden und bleibt entsprechend eine zukünftige Aufgabe, welche ausschließlich in einem konstruktiven Austausch zwischen Fachdidaktiken und allgemeiner Unterrichtsforschung gelingen kann.

Als Resümee lässt sich festhalten, dass der hier vorgenommene Fächervergleich einen wichtigen Ansatzpunkt darstellt, um unter dem Dach einer interdisziplinär ausgerichteten Bildungsforschung mögliche Gemeinsamkeiten und Unterschiede in Fächern systematisch zu identifizieren und fächervergleichende Forschungsaktivitäten voranzutreiben.

Funding Open access funding provided by University of Zurich 
Open Access Dieser Artikel wird unter der Creative Commons Namensnennung 4.0 International Lizenz veröffentlicht, welche die Nutzung, Vervielfältigung, Bearbeitung, Verbreitung und Wiedergabe in jeglichem Medium und Format erlaubt, sofern Sie den/die ursprünglichen Autor(en) und die Quelle ordnungsgemäß nennen, einen Link zur Creative Commons Lizenz beifügen und angeben, ob Änderungen vorgenommen wurden.

Die in diesem Artikel enthaltenen Bilder und sonstiges Drittmaterial unterliegen ebenfalls der genannten Creative Commons Lizenz, sofern sich aus der Abbildungslegende nichts anderes ergibt. Sofern das betreffende Material nicht unter der genannten Creative Commons Lizenz steht und die betreffende Handlung nicht nach gesetzlichen Vorschriften erlaubt ist, ist für die oben aufgeführten Weiterverwendungen des Materials die Einwilligung des jeweiligen Rechteinhabers einzuholen.

Weitere Details zur Lizenz entnehmen Sie bitte der Lizenzinformation auf http://creativecommons.org/ licenses/by/4.0/deed.de.

\section{Anhang}

\section{Einbezogene Literatur in Tab. 1 für die Fächergruppe Naturwissenschaften (geordnet nach den dazugehörigen Videostudien)}

\section{[1] A cross-cultural comparison (Biologie)}

- [1] Liu, N. \& Neuhaus, B. J. (2018). A cross-cultural comparison of biology lessons between China and Germany: a video study. Cultural Studies of Science Education. https://doi.org/10.1007/s11422-016-9797-6

[2] Bern Video Study (Physik)

- [2] Dalehefte, I. M., Rimmele, R., Prenzel, M., Seidel, T., Labudde, P., \& Herweg, C. (2009). Observing Instruction "next door": A Video Study about Science Teaching and Learning in Germany and Switzerland. In T. Janik, \& T. Seidel (Eds.), The Power of Video Studies in Investigating Teaching and Learning in the Classroom (pp. 83-101). Münster: Waxmann.

[3-4] Chemie in der Sekundarstufe I (Chemie)

- [3] Nehring, A., Päßler, A., \& Tiemann, R. (2015). The Complexity of Teacher Questions in Chemistry Classrooms: An Empirical Analysis on the Basis of Two Competence Models. International Journal of Science and Mathematics Education. https://doi.org/10.1007/s10763-015-9683-9

- [4] Puhlmann, M., \& Tiemann, R. (2010). Handlungsmuster und Problemlösen. Eine vergleichende Videostudie zwischen Nordrhein-Westfalen und Sachsen. In D. Höttecke (Hrsg.), Entwicklung naturwissenschaftlichen Denkens zwischen Phänomen und Systematik. Gesellschaft für Didaktik der Chemie und Physik Jahrestagung in Dresden 2009 (S. 434-436). Berlin: Lit Verlag.

[5-9] Dialogue I (Science)

- [5] Kiemer, K., Gröschner, A., Pehmer, A.-K., \& Seidel, T. (2014). Teacher learning and student outcomes in the context of classroom discourse. Findings from a video-based teacher professional development programme. Form@re - Open Journal per la formazione in rete, 14(2), 55-62. https://doi.org/10.13128/formare15124 
- [6] Kiemer, K., Gröschner, A., Pehmer, A.-K., \& Seidel, T. (2015). Effects of a classroom discourse intervention on teachers' practice and students' motivation to learn mathematics and science. Learning and Instruction, 35, 94-103. https:// doi.org/10.1016/j.learninstruc.2014.10.003

- [7] Pehmer, A.-K. (2015). A teacher intervention on students' higher-order learning in classroom dialogue. Dissertation: Technische Universität München.

- [8] Pehmer, A.-K., Gröschner, A., \& Seidel, T. (2015a). Fostering and scaffolding student engagement in productive classroom discourse: Teachers' practice changes and reflections in light of teacher professional development. Learning, Culture and Social Interaction, 7, 12-27. https://doi.org/10.1016/j.lcsi.2015.05.001

- [9] Pehmer, A.-K., Gröschner, A., \& Seidel, T. (2015b). How teacher professional development regarding classroom dialogue affects students' higher-order learning. Teaching and Teacher Education, 47, 108-119. https://doi.org/10.1016/j.tate.2014. 12.007

[10] Entwicklung und Evaluation einer Chemielehrerfortbildung zum Kompetenzbereich Erkenntnisgewinnung (Chemie)

- [10] Schmitt, A.-K. (2016). Entwicklung und Evaluation einer Chemielehrerfortbildung zum Kompetenzbereich Erkenntnisgewinnung. Studien zum Physik- und Chemielernen. In H. Niedderer, H. Fischler, \& E. Sumfleth (Hrsg.), Studien zum Physik- und Chemielernen. Band 198. Berlin: Logos Verlag.

[11] Fostering Conceptual Knowledge (Biologie)

- [11] Förtsch, C., Dorfner, T., Baumgartner, J., Werner, S., von Kotzebue, L., Heuhaus, J. N. (2018). Fostering Students' Conceptual Knowledge in Biology in the Context of German National Education Standards. Research in Science Education, 1-33. https://doi.org/10.1007/s11165-018-9709-8

[12] Instructional Quality Features in Videotaped Biology Lessons (Biologie)

- [12] Dorfner, T., Förtsch, C., Boone, W., Neuhaus, B. J. (2017b). Instructional Quality Featrues in Videotaped Biology Lessons: Content-Independent Description of Characteristics. Reasearch in Science Education (1-35). https://doi.org/10. 1007/s11165-017-9663-X

[13-38] Lehr-Lernprozesse im Physikunterricht - Eine Videostudie (IPN-Studie) (Physik)

- [13] Brückmann, M. (2009). Sachstrukturen im Physikunterricht: Ergebnisse einer Videostudie. In H. Niedderer, H. Fischler, \& E. Sumfleth (Hrsg.), Studien zu Physik- und Chemielernen. Band 94. Berlin: Logos Verlag

- [14] Dalehefte, I. M. (2006). Unterrichtsskripts - ein multikriterialer Ansatz. Eine Videostudie zum Zusammenspiel von Mustern unterrichtlicher Aktivitäten, Zielorientierung und prozessorientierter Lernbegleitung. Dissertation: Christian Albrechts Universität zu Kiel.

- [15] Herweg, C. (2008). Zielorientierung im deutschen und schweizerischen Physikunterricht - eine Videostudie -. Dissertation: Christian Albrechts Universität zu Kiel.

- [16] Knierim, B. (2007). Bedeutung von Lernbegleitung für die Entwicklung der Lernenden. In D. Höttecke (Hrsg.), Naturwissenschaftlicher Unterricht im internationalen Vergleich (S. 628-630). Berlin: Lit Verlag. 
- [17] Kobarg, M., \& Seidel, T. (2007). Prozessorientierte Lernbegleitung - Videoanalysen im Physikunterricht der Sekundarstufe I. Unterrichtswissenschaft, 35, $148-168$.

- [18] Labudde, P., \& Duit, R. (2007). Zum Design einer bi-nationalen Videostudie zum Physikunterricht. In D. Höttecke (Hrsg.), Naturwissenschaftlicher Unterricht im internationalen Vergleich (S. 631-633). Berlin: Lit Verlag.

- [19] Lehrke, M., Seidel, T., \& Prenzel, M. (2003). Dominierende Skripts im Physikunterricht und Unterrichtsergebnisse. In A. Pitton (Hrsg.), Außerschulisches Lernen in Physik und Chemie (S. 120-123). Münster: Lit Verlag. (GDCP-Tagungsband)

- [20] Meyer, L., Seidel, T., \& Prenzel, M. (2006). Wenn Lernsituationen zu Leistungssituationen werden: Untersuchung zur Fehlerkultur in einer Videostudie. Schweizerische Zeitschrift für Bildungswissenschaften, 28(1), 21-41.

- [21] Müller, C. T., \& Duit, R. (2004). Die unterrichtliche Sachstruktur als Indikator für Lernerfolg - Analyse von Sachstrukturdiagrammen und ihr Bezug zu Leistungsergebnissen im Physikunterricht. Zeitschrift für Didaktik der Naturwissenschaften, 10, 147-161.

- [22] Müller, C. T. (2004). Subjektive Theorien und handlungsleitende Kognitionen von Lehrern als Determinanten schulischer Lehr-Lern-Prozesse im Physikunterricht. In H. Niedderer, \& H. Fischler (Hrsg.), Studien zum Physiklernen. Band 33. Berlin: Logos Verlag.

- [23] Podschuweit, S., Bernholt, S., \& Brückmann, M. (2016). Classroom learning and achievement: How the complexity of classroom interaction impacts students' learning. Research in Science \& Technological Education, 34(2), 142-163. https:// doi.org/10.1080/02635143.2015.1092955

- [24] Prenzel, M., Seidel, T., Lehrke, M., Rimmele, R., Duit, R., Euler, M., Geiser, H., Hoffmann, L., Müller C., \& Widodo, A. (2002). Lehr-Lernprozesse im Physikunterricht - eine Videostudie. Zeitschrift für Pädagogik, 45. Beiheft, 139-156.

- [25] Seidel, T. (2003). Lehr-Lernskripts im Unterricht: Freiräume und Einschränkungen für kognitive und motivationale Lernprozesse; eine Videostudie im Physikunterricht. In D. H. Rost (Hrsg.), Pädagogische Psychologie und Entwicklungspsychologie. Band 35. Münster: Waxmann..

- [26] Seidel, T., \& Prenzel, M. (2004). Muster unterrichtlicher Aktivitäten im Physikunterricht. In J. Doll, \& M. Prenzel (Hrsg.), Bildungsqualität von Schule. Lehrerprofessionalisierung, Unterrichtsentwicklung und Schülerf.rderung als Strategien der Qualitätsverbesserung (S. 177-194). Münster: Waxmann.

- [27] Seidel, T., \& Prenzel, M. (2006). Stability of teaching patterns in physics instruction: Findings from a video study. Learning and Instruction, 16(3), 228-240. https://doi.org/10.1016/j.learninstruc.2006.03.002

- [28] Seidel, T., Prenzel, M., Duit, R., Euler, M., Geiser, H., Hoffmann, L., Lehrke, M., Müller, C. T., \& Rimmele, R. (2002). ,Jetzt bitte alle nach vorn schauen!“ - Lehr-Lernskripts im Physikunterricht und damit verbundenen Bedingungen für individuelle Lernprozesse. Unterrichtswissenschaft, 30(3), 52-77. 
- [29] Seidel, T., Prenzel, M., Rimmele, R., Dalehefte, I. M., Herweg, C., Kobarg, M., \& Schwindt, K. (2006a). Blicke auf den Physikunterricht. Ergebnisse der IPN Videostudie. Zeitschrift für Pädagogik, 52(6), 799-821.

- [30] Seidel, T., Prenzel, M., Rimmele, R., Herweg, C., Kobarg, M., Schwindt, K., \& Dalehefte, I. M. (2007). Science teaching and learning in German physics classrooms. Findings from the IPN Video Study. In M. Prenzel (Hrsg.), Studies on the educational quality of schools. The final report on the DFG priority programme (pp. 79-99). Münster: Waxmann.

- [31] Seidel, T., Prenzel, M., Rimmele, R., Schwindt, K., Kobarg, M., Herweg, C., \& Dalehefte, I. M. (2006b). Unterrichtsmuster und ihre Wirkungen. Eine Videostudie im Physikunterricht. In M. Prenzel, \& L. Allolio-Näcke (Hrsg.), Untersuchungen zur Bildungsqualität von Schule. Abschlussbericht des DFGSchwerpunktprogramms (S. 99-123). Münster: Waxmann.

- [32] Seidel, T., Prenzel, M., Schwindt, K., Rimmele, R., Kobarg, M., \& Dalehefte, I. M. (2009). The Link between Teaching and Learning-Investigating Effects of Physics Teaching on Student Learning in the Context of the IPN video Study. In T. Janik, \& T. Seidel (Eds.), The Power of Video Studies in Investigating Teaching and Learning in the Classroom (pp. 161-180). Münster: Waxmann.

- [33] Seidel, T., Rimmele, R., \& Prenzel, M. (2003). Gelegenheitsstrukturen beim Klassengespräch und ihre Bedeutung für die Lernmotivation. Videoanalysen in Kombination mit Schülerselbsteinsch.tzungen. Unterrichtswissenschaft, 31, $142-165$.

- [34] Seidel, T., Rimmele, R., \& Prenzel, M. (2005). Clarity and coherence of lesson goals as a scaffold for student learning. Learning and Instruction, 15(6), 539-556. https://doi.org/10.1016/j.learninstruc.2005.08.004

- [35] Tesch, M. (2005). Das Experiment im Physikunterricht: Didaktische Konzepte und Ergebnisse einer Videostudie. In H. Niedderer, H. Fischler, \& E. Sumfleth (Hrsg.), Studien zum Physik- und Chemielernen. Band 42. Berlin: Logos Verlag.

- [36] Tesch, M., \& Duit, R. (2004). Experimentieren im Physikunterricht - Ergebnisse einer Videostudie. Zeitschrift für Didaktik der Naturwissenschaften, 10, 51-69.

- [37] Tesch, M., \& Duit, R. (2005). Grundformen des Experimentierens. In A. Pitton (Hrsg.), Relevanz fachdidaktischer Forschungsergebnisse für die Lehrerbildung (S. 330-332). Münster: Lit Verlag. (GDCP-Tagungsband)

- [38] Widodo, A., \& Duit, R. (2004). Konstruktivistische Sichtweisen vom Lehren und Lernen und die Praxis des Physikunterrichts. Zeitschrift für Didaktik der Naturwissenschaften, 10, 233-255.

[39] Kompetenzorientierung und Aufgabenkultur im Natur-und-Technik-Unterricht (LerNT) (Biologie)

- [39] Förtsch, C., Werner, S., Dorfner, T., Kotzebue, L. von, \& Neuhaus, B. J. (2016). Effects of Cognitive Activation in Biology Lessons on Students' Situational Interest and Achievement. Research in Science Education. https://doi.org/ 10.1007/s11165-016-9517-y

[40] Nature of Science - Scientific Inquiry im internationalen Vergleich (Chemie) 
- [40] Stiller, J. (2016). Scientific Inquiry im Chemieunterricht - Eine Videoanalyse zur Umsetzung von Erkenntnisgewinnungsprozessen im internationalen und schulstufenübergreifenden Vergleich. Dissertation: Humboldt-Universität zu Berlin.

[41] nwu Essen (Videostudie Bildungswissenschaften) (Physik)

- [41] Schabram, K. (2007). Lernaufgaben im Unterricht: Instruktionspsychologische Analysen am Beispiel der Physik. Dissertation: Universität Duisburg-Essen. [42-51] nwu Essen (Videostudie Biologie) (Biologie)

- [42] Jatzwauk, P. (2007). Aufgaben im Biologieunterricht: Eine Analyse der Merkmale und des didaktischmethodischen Einsatzes von Aufgaben im Biologieunterricht. Berlin: Logos Verlag.

- [43] Jatzwauk, P., Rumann, S., \& Sandmann, A. (2008). Der Einfluss des Aufgabeneinsatzes im Biologieunterricht auf die Lernleistung der Schüler - Ergebnisse einer Videostudie. Zeitschrift für Didaktik der Naturwissenschaften, 14, 263-283.

- [44] Nawani, J., Rixius, J., \& Neuhaus, B. J. (2016). Influence of using challenging tasks in biology classrooms on students' cognitive knowledge structure: an empirical video study. International Journal of Science Education, 38(12), 1882-1903. https://doi.org/10.1080/09500693.2016.1213456

- [45] Nawani, J., Kotzebue, L., Rixius, J., Graml, M., Neuhaus, B. J. (2018). Teachers' Use of Focus Questions in German Biology Classrooms: a Video-based Naturalistic Study. International Journal of Science and Mathematics Education. https://doi.org/10.1007/s10763-017-9837-z

- [46] Wadouh, J. (2007). Vernetzung und kumulatives Lernen im Biologieunterricht der Gymnasialklasse 9. Dissertation: Universität Duisburg-Essen.

- [47] Wadouh, J., Liu, N., Sandmann, A., \& Neuhaus, B. J. (2014). The effect of knowledge linking levels in biology lessons upon students' knowledge structure. International Journal of Science and Mathematics Education, 12, 25-47.

- [48] Wadouh, J., Sandmann, A., \& Neuhaus, B. J. (2009). Vernetzung im Biologieunterricht - deskriptive Befunde einer Videostudie. Zeitschrift für Didaktik der Naturwissenschaften, 15, 69-87.

- [49] Wüsten, S. (2010). Allgemeine und fachspezifische Merkmale der Unterrichtsqualität im Fach Biologie. Eine Video- und Interventionsstudie. Berlin: Logos Verlag.

- [50] Wüsten, S., Schmelzing, S., Sandmann, A., \& Neuhaus, B. J. (2010). Fachspezifische Qualitätsmerkmale von Biologieunterricht. In U. Harms, \& I. Mackensen-Friedrichs (Hrsg.), Lehr- und Lernforschung in der Biologiedidaktik. Band 4. „Heterogenität erfassen - individuell fördern im Biologieunterricht“. Internationale Tagung der Fachsektion Didaktik der Biologie im VBIO, Kiel 2009 (S. 119-134). Innsbruck: StudienVerlag

- [51] Wüsten, S., Schmelzing, S., Sandmann, A., \& Neuhaus, B. (2010). Sachstrukturdiagramme - Eine Methode zur Erfassung inhaltsspezifischer Merkmale der Unterrichtsqualität im Biologieunterricht. Zeitschrift für Didaktik der Naturwissenschaften, 16, 23-39.

[52-53] nwu Essen (Videostudie Chemie I) (Chemie) 
- [52] Glemnitz, I. B. (2007). Vertikale Vernetzung im Chemieunterricht. Ein Vergleich von traditionellem Unterricht mit Unterricht nach Chemie im Kontext. In H. Niedderer, H. Fischler, \& E. Sumfleth (Hrsg.), Studien zum Physik- und Chemielernen. Band 62. Berlin: Logos Verlag.

- [53] Neumann, K., Fischer, H. E., \& Sumfleth, E. (2008). Vertikale Vernetzung und kumulatives lernen im Chemie- und Physikunterricht. In E.-M. Lankes (Hrsg.), Pädagogische Professionalität als Gegenstand empirischer Forschung (S. 141-151). Münster: Waxmann.

[54] nwu Essen (Videostudie Chemie II) (Chemie)

- [54] Schulz, A. (2011). Experimentierspezifische Qualitätsmerkmale im Chemieunterricht: Eine Videostudie. In H. Niedderer, H. Fischler, \& E. Sumfleth (Hrsg.), Studien zum Physik- und Chemielernen. Band 113. Berlin: Logos Verlag.

[55-57] nwu Essen (Videostudie Physik) (Physik)

- [55] Lau, A. (2011). Passung und vertikale Vernetzung im Chemie- und Physikunterricht. In. H. Niedderer, H. Fischler, \& E. Sumfleth (Hrsg.), Studien zum Physikund Chemielernen. Band 124. Berlin: Logos Verlag.

- [56] Lau, A., Neumann, K., Fischer, H. E., \& Sumfleth, E. (2009). Einfluss von Passung von Aufgaben und Antworten auf Schülerleistung. In D. Höttecke (Hrsg.), Chemie- und Physikdidaktik für die Lehramtsausbildung. Gesellschaft für Didaktik der Chemie und Physik Jahrestagung in Schwäbisch Gmünd 2008 (S. 304-306). Berlin: Lit Verlag.

- [57] Neumann, K., Fischer, H. E., \& Sumfleth, E. (2008). Vertikale Vernetzung und kumulatives lernen im Chemie- und Physikunterricht. In E.-M. Lankes (Hrsg.), Pädagogische Professionalität als Gegenstand empirischer Forschung (S. 141-151). Münster: Waxmann.

[58-62] nwu Essen (,PLUS-Projekt“) (Sachunterricht, Physik)

- [58] Ewerhardy, A. (2010). Zusammenhänge zwischen Verständnisorientierung von naturwissenschaftsbezogenem Sachunterricht und Fortschritten im Verständnis naturwissenschaftlicher Konzepte bei Lernenden der Grundschule. Dissertation: Westfälische Wilhelms-Universität Münster.

- [59] Ewerhardy, A., Kleickmann, T., \& Möller, K. (2012). Fördert ein konstruktivistisch orientierter naturwissenschaftlicher Sachunterricht mit strukturierenden Anteilen das konzeptuelle Verständnis bei den Lernenden? Zeitschrift für Grundschulforschung. Bildung im Elementar- und Primarbereich, 5(1), 76-88.

- [60] Fricke, K. (2016). Classroom Management and its Impact on Lesson Outcomes in Physics. A multiperspective comparison of teaching practices in primary and secondary schools. In H. Niedderer, H. Fischler, \& E. Sumfleth (Hrsg.), Studien zum Physik- und Chemielernen. Band 215. Berlin: Logos Verlag.

- [61] Ohle, A. (2010). Primary School Teachers' Content Knowledge in Physics and its Impact on Teaching and Students' Achievement. In H. Niedderer, H. Fischler, \& E. Sumfleth (Hrsg.), Studien zum Physik- und Chemielernen. Band 110. Berlin: Logos Verlag.

- [62] Ohle, A., Fischer, H. E., \& Kauertz, A. (2011). Der Einfluss des physikalischen Fachwissens von Primarstufenlehrkräften auf Unterrichtsgestaltung und Schülerleistung. Zeitschrift für Didaktik der Naturwissenschaften, 17, 357-389. 


\section{[63-64] Phi-Actio (Physik)}

- [63] Szogs, M., Krüger, M., Korneck, F. (2017). Erhebung von Unterrichtsqualität mittels hoch-inferenter Videorating - das Ratingmanual der Фactio-Studie. In Maurer, C. (Hrsg.) Implementation fachdidaktischer Innovation im Spiegel von Forschung und Praxis. Gesellschaft für Didaktik der Chemie und Physik Jahrestagung in Zürich 2016, 256-259.

- [64] Szogs, M., Korneck, F., Krüger, M., Oettinghaus, L., Kunter, M. (2016) Kognitive Aktivierung in standardisierten Unterrichtsminiaturen. In Maurer, C. (Hrsg.) Authentizität und Lernen - das Fach in der Fachdidaktik. Gesellschaft für Didaktik der Chemie und Physik Jahrestagung in Berlin 2015, 605-607.

[65] Practices of Science Observation (Science)

- [65] Forbes, C., Lange, K., Möller, K., Biggers, M., Laux, M., Zangori, L. (2014). Explanation-Construction in Fourth-Grade Classrooms in Germany and the USA: A cross-national comparative video study. International Journal of Science Education. https://doi.org/10.1080/09500693.2014.923950

[66-69] Professionswissen in den Naturwissenschaften - Teilprojekt Biologie (ProwiN) (Biologie)

- [66] Dorfner, T., Förtsch, C., Neuhaus, B. J. (2019). Use of technical terms in German biology lessons and ist effects on students' conceptual learning. Research in Science \& Technological Education. https://doi.org/10.1080/02635143.2019. 1609436

- [67] Förtsch, C., Werner, S., von Kotzebue, L., \& Neuhaus, B. J. (2016). Effects of Biology Teachers' Professional Knowledge and Cognitive Activation on Students' Achievement. International Journal of Science Education, 38 (17), 2642-2666. https://doi.org/10.1080/09500693.2016.1257170

- [68] Werner, S. (2016). Zusammenhänge zwischen dem fachspezifischen Professionswissen einer Lehrkraft, dessen Unterrichtsgestaltung und Schülervariablen am Beispiel eines elaborierten Modelleinsatzes. Dissertation: Ludwig-MaximiliansUniversität München

- [69] Werner, S., Förtsch, C., Boone, W., von Kotzebue, L. \& Neuhaus, B. J. (2017). Investigating how German biology teachers use models in classroom instruction: A video study. Research in Science Education. https://doi.org/10.1007/s11165017-9624-4

[70] Professionswissen in den Naturwissenschaften - Teilprojekt Chemie (ProwiN) (Chemie)

- [70] Strübe, M., Tröger, H., Tepner, O., \& Sumfleth, E. (2014). Development of a Pedagogical Content Knowledge test of chemistry language and models. Educación Química, 215(3), 380-390.

[71-72] Professionswissen in den Naturwissenschaften - Teilprojekt Physik (ProwiN) (Physik)

- [71] Cauet, E. (2015). Testen wir relevantes Wissen? Zusammenhänge zwischen dem Professionswissen von Physiklehrkräften und gutem und erfolgreichem Unterrichten. Dissertation: Universität Duisburg-Essen.

- [72] Lenske, G., Wagner, W., Wirth, J., Thillmann, H., Cauet, E., Liepertz, S., \& Leutner, D. (2016). Die Bedeutung des pädagogisch-psychologischen Wissens 
für die Qualität der Klassenführung und den Lernzuwachs der Schüler/innen im Physikunterricht. Zeitschrift für Erziehungswissenschaft, 19(1), 211-233. https:// doi.org/10.1007/s11618-015-0659-x

[73-84] Quality of Instruction in Physics (QuIP) (Physik)

- [73] Beerenwinkel, A., \& Arx, M. von. (2016). Constructivism in Practice: An Exploratory Study of Teaching Patterns and Student Motivation in Physics Classrooms in Finland, Germany and Switzerland. Research in Science Education. https://doi.org/10.1007/s11165-015-9497-3

- [74] Beerenwinkel, A., \& Börlin, J. (2014). Surface Level: Teaching Time, Lesson Phases and Types of Interaction. In H. E. Fischer, P. Labudde, K. Neumann, \& J. Viiri (Eds.), Quality of Instruction in Physics. Comparing Finland, Switzerland and Germany (pp. 65-79). Münster: Waxmann.

- [75] Börlin, J. (2012). Das Experiment als Lerngelegenheit: Vom interkulturellen Vergleich des Physikunterrichts zu Merkmalen seiner Qualität. In H. Niedderer, H. Fischler, \& E. Sumfleth (Hrsg.), Studien zum Physik- und Chemielernen. Band 132. Berlin: Logos Verlag.

- [76] Börlin, J., \& Labudde, P. (2014). Practical Work in Physics Instruction: An Opportunity to Learn? H. E. Fischer, P. Labudde, K. Neumann, \& J. Viiri (Eds.), Quality of Instruction in Physics. Comparing Finland, Switzerland and Germany (pp. 111-127). Münster: Waxmann.

- [77] Ergönenc, J., Neumann, K., \& Fischer, H. E. (2014). The Impact of Pedagogical Content Knowledge on Cognitive Activation and Student Learning. In H. E. Fischer, P. Labudde, K. Neumann, \& J. Viiri (Eds.), Quality of Instruction in Physics. Comparing Finland, Switzerland and Germany (pp. 145-159). Münster: Waxmann.

- [78] Geller, C., Neumann, K., \& Fischer, H. E. (2014). A Deeper Look inside Teaching Scripts: Learning Process Orientations in Finland, Germany and Switzerland. In H. E. Fischer, P. Labudde, K. Neumann, \& J. Viiri (Eds.), Quality of Instruction in Physics. Comparing Finland, Switzerland and Germany (pp. 81-92). Münster: Waxmann.

- [79] Helaakoski, J., \& Viiri, J. (2014). Content and Content Structure of Physics Lessons and Students' Learning Gains: Comparing Finland, Germany and Switzerland. In H. E. Fischer, P. Labudde, K. Neumann, \& J. Viiri (Eds.), Quality of Instruction in Physics. Comparing Finland, Switzerland and Germany (pp. 93-110). Münster: Waxmann.

- [80] Junge, C., Arx, M. von, \& Labudde, P. (2014). Classroom Management. In H. E. Fischer, P. Labudde, K. Neumann, \& J. Viiri (Eds.), Quality of Instruction in Physics. Comparing Finland, Switzerland and Germany (pp. 161-176). Münster: Waxmann.

- [81] Keller, M. M., Neumann, K., \& Fischer, H. E. (2014). Enthusiastic Teaching and its Impact on Students' Interest and Self-Concept: An Investigation of German Physics Classrooms. In H. E. Fischer, P. Labudde, K. Neumann, \& J. Viiri (Eds.), Quality of Instruction in Physics. Comparing Finland, Switzerland and Germany (pp. 129-143). Münster: Waxmann. 
- [82] Keller, M. M., Neumann, K., Fischer, H. E. (2017). The Impact of of Physics Teachers' Pedagogical Content Knowledge and Motivation on Students' Achievement and Interest. Journal of Reasearch in Science Teaching. https://doi.org/10. $1002 /$ tea. 21378

- [83] Olszewski, J. (2010). The impact of physics teachers' pedagogical content knowledge on teacher action and student outcomes. In H. Niedderer, H. Fischler, \& E. Sumfleth (Hrsg.), Studien zum Physik- und Chemielernen. Band 109. Berlin: Logos Verlag.

- [84] von Arx, M. (2014). Constructivist Approaches to Teaching. In H. E. Fischer, P. Labudde, K. Neumann, \& J. Viiri (Eds.), Quality of Instruction in Physics. Comparing Finland, Switzerland and Germany (pp. 177-192). Münster: Waxmann.

[85] SINUS an Grundschulen (Sachunterricht)

- [85] Kobarg, M., Dalehefte, I. M., \& Menk, M. (2012). Der Einsatz systematischer Videoanalysen zur Untersuchung der Wirksamkeit des Unterrichtsentwicklungsprogramms „SINUS an Grundschulen“. In M. Kobarg, C. Fischer, I. M. Dalehefte, F. Trepke, \& M. Menk (Hrsg.), Lehrerprofessionalisierung wissenschaftlich begleiten. Strategien und Methoden (S. 181-194). Münster: Waxmann.

[86] Teaching quality and metacognitive strategy use (Science)

- [86] Rieser, S., Naumann, A., Decristan, J., Fauth, B., Klieme, E., Büttner, G. (2016). The connection between teaching and learning: Linking teaching quality and metacognitive strategy use in primary school. British Journal of Educational Psychology, 86, 526-545. https://doi.org/10.1111/bjep.12121

[87-89] Überprüfung der Wirksamkeit eines Basismodell-Trainings für Physiklehrer (Physik)

- [87] Wackermann, R. (2008). .berprüfung der Wirksamkeit eines BasismodellTrainings für Physiklehrer. In H. Niedderer, H. Fischler, \& E. Sumfleth (Hrsg.), Studien zum Physik- und Chemielernen. Band 75. Berlin: Logos Verlag.

- [88] Wackermann, R., Trendel, G., \& Fischer, H. E. (2010). Evaluation of a Theory of Instructional Sequences for Physics Instruction. International Journal of Science Education, 32(7), 963-985. https://doi.org/10.1080/09500690902984792

- [89] Trendel, G., Wackermann, R., \& Fischer, H. E. (2007). Lernprozessorientierte Lehrerfortbildung in Physik. Zeitschrift für Didaktik der Naturwissenschaften, 13, 9-31.

[90-91] Unterrichtsgestaltung und Lernerfolg im Physikunterricht (Physik)

- [90] Fischer, H. E., Reyer, T., Wirz, C., Bos, W., \& Höllrich, N. (2002). Unterrichtsgestaltung und Lernerfolg im Physikunterricht. Zeitschrift für Pädagogik, 124-138.

- [91] Reyer, T. (2004). Oberflächenmerkmale und Tiefenstrukturen im Unterricht. Exemplarische Analysen im Physikunterricht der gymnasialen Sekundarstufe. In H. Niedderer, \& H. Fischler (Hrsg.), Studien zum Physiklernen. Band 32. Berlin: Logos Verlag.

[92-94] Veränderung des fachspezifisch-pädagogischen Wissens bei Grundschullehrkräften in Bezug auf Lehren und Lernen im naturwissenschaftlichen Unterricht durch Lehrerfortbildungen (Sachunterricht) 
- [92] Kleickmann, T., Tröbst, S., Jonen, A., Vehmeyer, J., \& Möller, K. (2016). The effects of expert scaffolding in elementary science professional development on teachers' beliefs and motivations, instructional practices, and student achievement. Journal of Educational Psychology, 108(1), 21-42. https://doi.org/10.1037/ edu0000041

- [93] Kleickmann, T., Vehmeyer, J., \& Möller, K. (2010). Zusammenhänge zwischen Lehrervorstellungen und kognitivem Strukturieren im Unterricht am Beispiel von Scaffolding-Maßnahmen. Unterrichtswissenschaft, 38(3), 210-228.

- [94] Vehmeyer, J. (2009). Kognitiv anregende Verhaltensweisen von Lehrkräften im naturwissenschaftlichen Sachuntericht - Konzeptualisierung und Erfassung. Dissertation: Westfälische Wilhelms-Universität Münster.

[95] VerE-Projekt (Biologie, Chemie)

- [95] Nehring, A., Stiller, J., Nowak, K. H., Upmeier zu Belzen, A., \& Tiemann, R. (2016). Naturwissenschaftliche Denk- und Arbeitsweisen im Chemieunterricht - eine modellbasierte Videostudie zu Lerngelegenheiten für den Kompetenzbereich der Erkenntnisgewinnung. Zeitschrift für Didaktik der Naturwissenschaften, 22(1), 77-96. https://doi.org/10.1007/s40573-016-0043-2

\section{Einbezogene Literatur in Tab. 1 für das Fach Sport}

[1] Balz, E. (2010). Guter Sportunterricht - Merkmale und Beispiele. sportpädagogik, 34, 50-53.

[2] Herrmann, C. (2019). Evaluation der Unterrichtsqualität im Sportunterricht mit dem QUALLIS-Instrument. Bewegung \& Sport, 12-17.

[3] Niederkofler, B. \& Amesberger, G. (2016). Kognitive Handlungsrepräsentationen als Strukturgrundlage zur Definition von kognitiver Aktivierung im Sportunterricht. Sportwissenschaft, 46, 188-200.

[4] Herrmann, C., Seiler, S. \& Niederkofler, B. (2016). „Was ist guter Sportunterricht?“ - Dimensionen der Unterrichtsqualität. sportunterricht, 65, 7-12.

[5] Gebken, U. (2005). Guter Sportunterricht für alle! In A. Gogoll \& A. MenzeSonneck (Hrsg.), Qualität im Schulsport (S. 234-239). Hamburg: Czwalina.

[6] Herrmann, C. \& Gerlach, E. (2017). Unterrichtsqualität im Schneesport. In I. Bach (Hrsg.), Skilauf und Snowboard in Lehre und Forschung (Band 24, S. 118-129). Hamburg: Czwalina.

[7] Linka, T. \& Gerlach, E. (2019). Klassenmanagement im Sportunterricht. In M. Hartmann, R. Laging \& C. Scheinert (Hrsg.), Professionalisierung in der Sportlehrer*innenbildung. (S. 95-107). Baltmannsweiler: Schneider.

[8] Steinegger, A. (2013). Prozessmerkmale guten Sportunterrichts. In R. Messmer (Hrsg.), Fachdidaktik Sport (S. 188-196). Bern: UTB.

[9] Laging, R. (2016). Bewegungsaufgaben als ,ästhetische Aktivierung ‘ - ein Beitrag zur professionstheoretischen Einordnung der Aufgabenforschung. In D. Wiesche, M. Fahlenbock \& N. Gissel (Hrsg.), Sportpädagogische Praxis (S. 251-259). Hamburg: Feldhaus.

[10] Wiesche, D. \& Klinge, A. (Hrsg.). (2017). Scham und Beschämung im Schulsport. Aachen: Meyer \& Meyer. 
[11] Wolters, P. \& Kemna, P. (2011). Qualitätskriterien für den Sportunterricht - Entwurf eines Rahmenmodells und einer Skala zur erlebten Sicherheit. In K.O. Bauer \& N. Logemann (Hrsg.), Unterrichtsqualität und fachdidaktische Forschung. (S. 159-186). Münster: Waxmann.

[12] Bundesamt für Sport. (Hrsg.). (2010). qims.ch. Qualität im Sport- und Bewegungsunterricht. Magglingen: BASPO.

[13] Baumgartner, M. (2018). Performanzfortschritt in der Lehrerausbildung durch die Arbeit an eigenen video- und textbasierten Unterrichtsfällen? Zeitschrift für Erziehungswissenschaft, 21, 1135-1155.

[14] Gerlach, E. (2005). Prima Klima? Einflussgrößen und Effekte. sportunterricht, $54,243-247$.

[15] Heemsoth, T. \& Miethling, W.-D. (2012). Schülerwahrnehmungen des Unterrichtsklimas. Entwicklung eines Fragebogens und Befund zum Sportunterricht. Sportwissenschaft, 42, 228-239.

[16] Egger, K., Kühnis, J. B., Nussbaum, P. \& Däniken, P. (2002). Qualität des Sportunterrichts. Magglingen: BASPO.

[17] Herrmann, C., Seiler, S., Pühse, U. \& Gerlach, E. (2015). „Wie misst man guten Sportunterricht?" - Erfassung der Dimensionen von Unterrichtsqualität im Schulfach Sport. Zeitschrift für Sportpädagogische Forschung, 3(1), 6-26.

[18] Niederkofler, B., Herrmann, C., Seiler, S. \& Gerlach, E. (2015). What influences Motivation in Physical Education? A Multilevel Approach to Identify Climate Determinants of Achievement Motivation. Psychological Test and Assessment Modeling, 57(1), 70-93.

[19] Büchel, S. (2019). Lehrermotivation im Sportunterricht. Wiesbaden: Springer. [20] Heemsoth, T. (2014). Unterrichtsklima als Mediator des Zusammenhangs von Klassenführung und Motivation im Sportunterricht. Psychologie in Erziehung und Unterricht, 61, 203-215.

[21] Heemsoth, T. \& Krieger, C. (2018). Perspektiven auf Sportunterricht. Unterrichtswissenschaft, 46, 499-522.

[22] Erdtel, M. \& Hummel, A. (2005). Qualitätsentwicklung im Schulsport - Möglichkeiten und Grenzen der Evaluierung von Qualität im Sportunterricht im Rahmen quantitativer Schulsportstudien. In A. Gogoll \& A. Menze-Sonneck (Hrsg.), Qualität im Schulsport (S. 48-53). Hamburg: Czwalina.

[23] Adler, K., Erdtel, M. \& Hummel, A. (2006). Belastungszeit und Belastungsintensität als Kriterien der Qualität im Sportunterricht? sportunterricht, 55, 45-49.

[24] Hoffmann, A. (2011). Bewegungszeit als Qualitätskriterium des Sportunterrichts. Spectrum der Sportwissenschaften, 23(1), 25-51.

[25] Hummel, A. \& Adler, K. (2005). Bewegungsdauer und Bewegungsintensität Gütekriterien eines guten Sportunterrichts? Chemnitz: TU.

[26] Kühnis, J., Eckert, N., Mandel, D., Imholz, P., Egli, S., Steffan, M. et al. (2017). Zeitnutzung und Anstrengung im Sportunterricht. Befunde einer Querschnittsstudie auf der Primarstufe im Kanton Schwyz. Swiss Sports \& Exercise Medicine, 65(3), 54-59.

[27] Neumann, P. \& Hafner, S. (2012). Bewegungslernzeit anbieten! sportpädagogik, $36(2), 2-7$. 
[28] Wydra, G. (2009). Belastungszeiten und Anstrengung im Sportunterricht. sportunterricht, 58, 129-136.

\section{Einbezogene Literatur in Tab. 1 für das Fach Geschichte}

[1] Gautschi, P. (2015): Guter Geschichtsunterricht. Grundlagen, Erkenntnisse (Geschichtsunterricht erforschen, Bd. 1), Hinweise. 3., Schwalbach 2015: Wochenschau Verlag (erste Auflage 2009).

[2] Reisman, A. (2011): Reading like a Historian: A Document-based History Curriculum Intervention in Urban High Schools.

[3] Reisman, A. (2012a): "Reading like a historian:" A document-based history curriculum intervention in urban high schools. In: Cognition and Instruction 30, 86-112. (x)

[4] Reisman, A. (2012b): "Text Complexity" in the history classroom: Teaching to and beyond the Common Core. In: Social Studies Review 51.

[5] Reisman, A. (2012c): The "Document-Based Lesson": Bringing Disciplinary Inquiry into High School History Classrooms with Adolescent Struggling Readers. In: Journal of Curriculum Studies 44, 233-264.

[6] Cohors-Fresenborg, E., Kaune, C., Zülsdorf-Kersting, M. (2014): Klassifikation von metakognitiven und diskursiven Aktivitäten im Mathematik- und Geschichtsunterricht. mit einem gemeinsamen Kategoriensystem (Schriftenreihe des Forschungsinstituts für Mathematikdidaktik, Bd. 63). Osnabrück: Forschungsinstitut für Mathematikdidaktik.

[7] Zülsdorf-Kersting, M., Praetorius, A.-K. (2017): Geschichtsunterricht zuverlässig beurteilen. Vorstellung eines Beobachtungsinstruments zur Bestimmung von metakognitiv-diskursiver Unterrichtsqualität. In: Zeitschrift für Geschichtsdidaktik 16, 250-265.

[8] Zülsdorf-Kersting, M. (2018a): Geschichtsunterricht als soziales System. In: Bracke, S., Flaving, C., Jansen, J., Köster, M., Lahmer-Gebauer, J., Lankes, S., Spieß, C., Thünemann, H., Wilfert, C., Zülsdorf-Kersting, M. (2018): Theorie des Geschichtsunterrichts (Geschichtsunterricht erforschen, Bd. 9). Frankfurt a. M., S. 13-69: Wochenschau Verlag.

[9] Maggioni, L. (2010): Studying epistemic Cognition in the History Classroom: Cases of Teaching and Learning to think historically. Dissertation submitted to the Faculty of the Graduate School of the University of Maryland, College Park, in partial fulfillment of the requirements for the degree of Doctor of Philosophy.

[10] Gautschi, P., Moser, D., Reusser, K., Wiher, P. (Hrsg.). (2007): Geschichtsunterricht heute. Eine empirische Analyse ausgewählter Aspekte. Bern: hep-Verlag.

[11] Köster, M., Thünemann, H., Zülsdorf-Kersting, M. (2017): Geschichtsunterricht als soziales System zwischen Angebot und Nutzung. In: Zeitschrift für Geschichtsdidaktik 16, 210-226: Vandenhoeck Ruprecht.

[12] Mägdefrau, J., Michler, A., Böhm, M., Jonas, K. (2017): Lernstrategieeinsatz von Schülern im Geschichtsunterricht - Aufgabenbezogene Erfassung der Nutzung 
kognitiver Lernstrategien im Schüler-Experten-Vergleich. In: Empirische Pädagogik 31: Verlag Empirische Pädagogik.

[13] Mägdefrau, J., Michler, A. (2012): Individualisierende Lernaufgaben im Geschichtsunterricht. Eine empirische Untersuchung zur Rolle von Schulbuchaufgaben und Eigenkonstruktionen der Lehrkräfte. In: Zeitschrift für Geschichtsdidaktik 11 208-232: Vandenhoeck Ruprecht.

[14] Martens, M. (2010): Implizites Wissen und kompetentes Handeln. Die empirische Rekonstruktion von Kompetenzen historischen Verstehens im Umgang mit Darstellungen von Geschichte (Beihefte zur Zeitschrift für Geschichtsdidaktik, Bd. 1). Göttingen: V\&R unipress.

[15] Pandel, H.-J. (2013): Geschichtsdidaktik. Eine Einführung. Schwalbach/Ts.: Wochenschau Verlag.

[16] Schönemann, B., Thünemann, H., Zülsdorf-Kersting, M. (2011): Was können Abiturienten? Zugleich ein Beitrag zur Debatte über Kompetenzen und Standards im Fach Geschichte (Geschichtskultur und historisches Lernen, Bd. 4). 2. Aufl. Berlin 2011: Lit Verlag.

[17] Spieß, C. (2014): Quellenarbeit im Geschichtsunterricht. Die empirische Rekonstruktion von Kompetenzerwerb im Umgang mit Quellen (Beihefte zur Zeitschrift für Geschichtsdidaktik, Bd. 8). Göttingen: Vandenhoeck Ruprecht.

\section{Literatur}

Bachleitner, R., Weichbold, M., Aschauer, W., \& Pausch, M. (2014). Methodik und Methodologie interkultureller Umfrageforschung. Wiesbaden: Springer VS.

Bracke, S., Flaving, C., Jansen, J., Köster, M., Lahmer-Gebauer, J., Lankes, S., et al. (2018). Theorie des Geschichtsunterrichts. Frankfurt a.M.: Wochenschau Verlag.

Bybee, R. W. (2006). Scientific inquiry and science teaching BT-Scientific inquiry and nature of science: implications for teaching, learning, and teacher education. In L. B. Flick \& N. G. Lederman (Hrsg.), Scientific inquiry and nature of science (S. 1-14). Dordrecht: Springer Netherlands.

Charalambous, C. Y., \& Litke, E. (2018). Studying instructional quality by using a content-specific lens: the case of the mathematical quality of instruction framework. ZDM Mathematics Education, 50(3), $445-460$.

Charalambous, C. Y., \& Praetorius, A.-K. (2020). Creating a forum for researching teaching and its quality more synergistically. Studies in Educational Evaluation. https://doi.org/10.1016/j.stueduc.2020. 100894. (online first)

Cohors-Fresenborg, E., Kaune, C., \& Zülsdorf-Kersting, M. (2014). Klassifikation von metakognitiven und diskursiven Aktivitäten im Mathematik- und Geschichtsunterricht mit einem gemeinsamen Kategoriensystem. Osnabrück: Forschungsinstitut für Mathematikdidaktik.

Dorfner, T., Förtsch, C., \& Neuhaus, B. J. (2017). Die methodische und inhaltliche Ausrichtung quantitativer Videostudien zur Unterrichtsqualität im mathematisch-naturwissenschaftlichen Unterricht. Zeitschrift für Didaktik der Naturwissenschaften, 23(1), 261-285. https://doi.org/10.1007/s40573-0170058-3

Helmke, A. (2017). Unterrichtsqualität und Lehrerprofessionalität. Diagnose, Evaluation und Verbesserung des Unterrichts. Bd. 7. Seelze: Klett.

Klieme, E., Schümer, G., \& Knoll, S. (2001). Mathematikunterricht in der Sekundarstufe I. „Aufgabenkultur" und Unterrichtsgestaltung. In E. Klieme \& J. Baumert (Hrsg.), TIMSS - Impulse für Schule und Unterricht. Forschungsbefunde, Refominitiativen, Prasixberichte und Video-Dokumente (S. 43-57). Bonn: Bundesministerium für Bildung und Forschung.

Kunter, M., \& Ewald, S. (2016). Bedingungen und Effekte von Unterricht: Aktuelle Forschungsperspektiven aus der pädagogischen Psychologie. In N. McElvany, W. Bos, H. G. Holtappels, M. M. Gebauer \& F. Schwabe (Hrsg.), Bedingungen und Effekte guten Unterrichts (S. 9-31). Münster: Waxmann. 
Kunter, M., \& Trautwein, U. (2013). Psychologie des Unterrichts. Paderborn: Ferdinand Schöningh.

Learning Mathematics for Teaching Project (2011). Measuring the mathematical quality of instruction. Journal of Mathematics Teacher Education, 14(1), 25-47.

Martin-Raugh, M., Tannenbaum, R. J., Tocci, C. M., \& Reese, C. (2016). Behaviorally anchored rating scales: An application for evaluating teaching practice. Teaching and Teacher Education, 59, 414-419.

Muijs, D., Kyriakides, L., van der Werf, G., Creemers, B., Timperley, H., \& Earl, L. (2014). State of the art-Teacher effectiveness and professional learning. School Effectiveness and School Improvement, 25(2), 231-256.

Neumann, K. (2018). Unterrichtsqualität in den Naturwissenschaften - Die Suche nach dem Heiligen Gral. In C. Maurer (Hrsg.), Qualitätsvoller Chemie- und Physikunterricht - normative und empirische Dimensionen. Gesellschaft für Didaktik der Chemie und Physik Jahrestagung, Regensburg, 2017. (S. 5-18).

Neumann, P., \& Balz, E. (Hrsg.). (2013). Sportdidaktik. Berlin: Cornelsen.

Niederkofler, B., \& Amesberger, G. (2016). Kognitive Handlungsrepräsentationen als Strukturgrundlage zur Definition von kognitiver Aktivierung im Sportunterricht. Sportwissenschaft, 46, 188-200.

Nowińska, E. (2016). Leitfragen zur Analyse und Beurteilung metakognitiv-diskursiver Unterrichtsqualität. Osnabrück: Forschungsinstitut für Mathematikdidaktik.

Openshaw, K., \& Clarke, S.C.T. (1970). General teaching theory. Journal of Teacher Education, 21(3), 403-416.

Praetorius, A.-K., \& Charalambous, C. Y. (2018). Classroom observation frameworks for studying instructional quality: looking back and looking forward. ZDM Mathematics Education, 50(3), 535-553.

Praetorius, A.-K., Klieme, E., Herbert, B., \& Pinger, P. (2018). Generic dimensions of teaching quality: the German framework of three basic dimensions. ZDM Mathematics Education, 50(3), 407-426.

Reusser, K. (2008). Empirisch fundierte Didaktik - didaktisch fundierte Unterrichtsforschung. Zeitschrift für Erziehungswissenschaft, Sonderheft 9, 219-237.

Rippl, S., \& Seipel, C. (2008). Methoden kulturvergleichender Sozialforschung. Wiesbaden: VS.

Risch, B., \& Peifer, P. (2018). Didaktische Reduktion - Elementarisierung. In K. Sommer, J. WambachLaicher \& P. Peifer (Hrsg.), Konkrete Fachdidaktik Chemie. Grundlagen für das Lernen und Lehren im Chemieunterricht (S. 45-63). Seelze: Aulis.

Schoenfeld, A.H. (2018). Video analyses for research and professional development: the teaching for robust understanding (TRU) framework. ZDM Mathematics Education, 50(3), 491-506.

Schulz, A. (2011). Experimentierspezifische Qualitätsmerkmale im Chemieunterricht: Eine Videostudie. In H. Niedderer, H. Fischler \& E. Sumfleth (Hrsg.), Studien zum Physik- und Chemielernen Bd. 113. Berlin: Logos.

Seidel, T., \& Shavelson, R. J. (2007). Teaching effectiveness research in the past decade. The role of theory and research design in disentangling meta-analysis results. Review of Educational Research, 77(4), 454-499.

Vieluf, S., Praetorius, A.-K., Rakoczy, K., Kleinknecht, M., \& Pietsch, M. (2020). Angebots-Nutzungsmodelle der Wirkweise des Unterrichts: eine kritische Auseinandersetzung mit ihrer theoretischen Konzeption. Zeitschrift für Pädagogik, 66(Beiheft 1/20), 63-80.

Ziegler, A., Antes, G. \& König, I. (2011). Bevorzugte Report Items für systematische Übersichten und Meta-Analysen: Das PRISMA-Statement. Deutsche Medizinische Wochenschrift, 136(8), e9-e15. 